\title{
Intralimb and Interlimb Cutaneous Reflexes during Locomotion in the Intact Cat
}

\author{
Marie-France Hurteau, ${ }^{1}$ Yann Thibaudier, ${ }^{1}$ Charline Dambreville, ${ }^{1}$ Simon M. Danner, ${ }^{2}$ Ilya A. Rybak, ${ }^{2}$ \\ and ${ }^{\circledR}$ Alain Frigon ${ }^{1}$ \\ ${ }^{1}$ Department of Pharmacology-Physiology, Faculty of Medicine and Health Sciences, Université de Sherbrooke, Sherbrooke, Quebec J1H 5N4, Canada, \\ and ${ }^{2}$ Department of Neurobiology and Anatomy, Drexel University College of Medicine, Philadelphia, Pennsylvania 19129
}

When the foot contacts an obstacle during locomotion, cutaneous inputs activate spinal circuits to ensure dynamic balance and forward progression. In quadrupeds, this requires coordinated reflex responses between the four limbs. Here, we investigated the patterns and phasic modulation of cutaneous reflexes in forelimb and hindlimb muscles evoked by inputs from all four limbs. Five female cats were implanted to record muscle activity and to stimulate the superficial peroneal and superficial radial nerves during locomotion. Stimulating these nerves evoked short-, mid-, and longer-latency excitatory and/or inhibitory responses in all four limbs that were phasedependent. The largest responses were generally observed during the peak activity of the muscle. Cutaneous reflexes during mid-swing were consistent with flexion of the homonymous limb and accompanied by modification of the stance phases of the other three limbs, by coactivating flexors and extensors and/or by delaying push-off. Cutaneous reflexes during mid-stance were consistent with stabilizing the homonymous limb by delaying and then facilitating its push-off and modifying the support phases of the homolateral and diagonal limbs, characterized by coactivating flexors and extensors, reinforcing extensor activity and/or delaying push-off. The shortest latencies of homolateral and diagonal responses were consistent with fast-conducting disynaptic or trisynaptic pathways. Descending homolateral and diagonal pathways from the forelimbs to the hindlimbs had a higher probability of eliciting responses compared with ascending pathways from the hindlimbs to the forelimbs. Thus, in quadrupeds, intralimb and interlimb reflexes activated by cutaneous inputs ensure dynamic coordination of the four limbs, producing a whole-body response.

Key words: cutaneous; interlimb coordination; locomotion; reflexes

\section{Significance Statement}

The skin contains receptors that, when activated, send inputs to spinal circuits, signaling a perturbation. Rapid responses, or reflexes, in muscles of the contacted limb and opposite homologous limb help maintain balance and forward progression. Here, we investigated reflexes during quadrupedal locomotion in the cat by electrically stimulating cutaneous nerves in each of the four limbs. Functionally, responses appear to modify the trajectory or stabilize the movement of the stimulated limb while modifying the support phase of the other limbs. Reflexes between limbs are mediated by fast-conducting pathways that involve excitatory and inhibitory circuits controlling each limb. The comparatively stronger descending pathways from cervical to lumbar circuits controlling the forelimbs and hindlimbs, respectively, could serve a protective function.

\section{Introduction}

During terrestrial locomotion, the limbs of bipeds and quadrupeds are coordinated to maintain dynamic balance in the envi-

Received Nov. 14, 2017; revised March 3, 2018; accepted March 9, 2018.

Author contributions: M.-F.H. and A.F. designed research; M.-F.H., Y.T., C.D., and A.F. performed research; M.-F.H., S.M.D., I.A.R., and A.F. analyzed data; M.-F.H., Y.T., C.D., S.M.D., I.A.R., and A.F. wrote the paper.

This work was supported by Natural Sciences and Engineering Research Council of Canada Discovery Grant RGPIN-2016-03790, Fonds de Recherche du Québec-Natures et Technologies Project Team Grant 182035, and Arthur C. Guyton Award for Excellence in Integrative Physiology. A.F. was supported by American Physiological Society Beverly Petterson Bishop Award for Excellence in Neuroscience. I.A.R. was supported by National Institutes of Health Grant R01 NS090919. M.-F.H. was supported by Natural Sciences and Engineering Research Council of Canada ronment and to accomplish goal-oriented behaviors. When a limb movement is perturbed, such as when contacting an obstacle, coordinated reflex responses ensure dynamic balance of the body while progressing forward. For example, mechanically or doctoral scholarship. We thank Philippe Drapeau (Rossignol and Drew labs, Université de Montréal) for developing and providing the data acquisition and analysis software.

The authors declare no competing financial interests.

Correspondence should be addressed to Dr. Alain Frigon, Université de Sherbrooke, 3001, 12e Avenue Nord, Department of Pharmacology-Physiology, Faculty of Medicine and Health Sciences, Sherbrooke, Quebec J1H 5N4, Canada. E-mail: alain.frigon@usherbrooke.ca.

DOI:10.1523/JNEUROSCI.3288-17.2018

Copyright $\odot 2018$ the authors $\quad 0270-6474 / 18 / 384104-19 \$ 15.00 / 0$ 
electrically stimulating the foot dorsum or superficial peroneal (SP) nerve during the swing phase elicits a coordinated reflex response, termed the stumbling corrective reaction, that lifts the leg away and over the stimulus while reinforcing support of the contralateral homologous limb in cats (Forssberg et al., 1977; Prochazka et al., 1978; Forssberg, 1979; Duysens and Loeb, 1980; Buford and Smith, 1993; Quevedo et al., 2005a) and humans (Schillings et al., 1996, 2000, 2005; Zehr et al., 1997; Haridas and Zehr, 2003). The stumbling corrective reaction is observed in cats with a spinal transection (spinal cats), indicating that spinal circuits are sufficient to generate the response (Forssberg et al., 1977; Frigon and Rossignol, 2008a; Hurteau et al., 2017). Similarly, mechanically or electrically stimulating the forepaw dorsum, or the superficial radial (SR) nerve that supplies it, during the forelimb swing phase in intact or decerebrate cats during quadrupedal locomotion elicits a pattern consistent with stumbling corrective reaction (Miller et al., 1977; Matsukawa et al., 1982; Drew and Rossignol, 1985, 1987; Fuwa et al., 1991). The reflex responses evoked by mechanical or electrical stimulation of the paw dorsa or their nerves are phase-dependent so that the same stimuli applied during stance prevents limb flexion, which has been termed a stumbling preventive reaction (Quevedo et al., 2005b).

Evidently, during quadrupedal locomotion, all four limbs contribute to dynamic balance. However, patterns of reflex responses evoked by stimulating cutaneous inputs in the forelimbs with recordings in the hindlimbs, and vice versa, have received little attention during quadrupedal locomotion (for human bipedal locomotion, see Dietz et al., 2001; Haridas and Zehr, 2003; Lamont and Zehr, 2006, 2007; Zehr and Loadman, 2012). Miller et al. (1977) observed responses in two homolateral forelimb and hindlimb muscles evoked by stimulating the skin or nerves of the hindpaw and forepaw dorsum, respectively, during treadmill locomotion in decerebrate cats. These homolateral responses were phase-dependent, suggesting that they could be functionally important for interlimb coordination during locomotion. However, responses evoked in diagonal limbs were not investigated, despite the fact that cats preferentially trot, which is characterized by diagonal limb coupling (Hildebrand, 1989). Anatomical studies in cats and rats also showed that long propriospinal pathways, which are thought to mediate interlimb reflexes between the forelimbs and hindlimbs, have denser projections to the circuits controlling diagonal limbs compared with those controlling the homolateral limb (English et al., 1985; Reed et al., 2006; Brockett et al., 2013; Ruder et al., 2016).

Thus, despite the work of Miller et al. (1977) with cutaneous stimulation in decerebrate cats, important gaps in knowledge concerning limb coordination during stumbling remain. First, it is important to understand whether ascending and descending interlimb reflexes, both homolateral and diagonal, are present during locomotion and whether they are phase-dependent in intact cats. Unfortunately, reflexes between the forelimbs and hindlimbs have not been investigated in an intact animal model. This is surprising because interlimb reflexes could be a powerful tool to study how pathways that coordinate the limbs are controlled and reorganized after neurological injury. Second, it is not clear whether patterns of evoked responses in flexors and extensors are similar to those of homonymous (in the stimulated limb) and crossed (in the contralateral homologous limb) responses. Such a comparison would allow us to determine which circuits are activated by cutaneous inputs in all four limbs. In addition, analyzing the latencies of these responses would allow us to estimate the number of synaptic relays in the pathways. Therefore, the main goal of this study was to characterize and compare reflex responses evoked by cutaneous inputs from all four limbs in several forelimb and hindlimb muscles in intact cats during treadmill locomotion.

\section{Materials and Methods}

Ethical approval. The procedures were performed according to the policies and directives of the Canadian Council on Animal Care, and all protocols were approved by the Animal Care Committee of the Université de Sherbrooke (Protocol 273-15). Experiments were performed on 5 female cats weighing between 3.5 and $5.0 \mathrm{~kg}$. As part of our effort to maximize the scientific output of each animal, some of the cats used in the present study were used in other projects to answer different questions, some of which have been published (Frigon et al., 2013, 2014; Thibaudier et al., 2013, 2017; D’Angelo et al., 2014; Thibaudier and Frigon, 2014; Kuczynski et al., 2017).

Implantation procedure. The implantation of electrodes for EMG and nerve stimulation was performed under aseptic conditions in an operating room with sterilized equipment, as described by Hurteau et al. (2017). Before surgery, sedation was induced by an intramuscular injection of butorphanol $(0.4 \mathrm{mg} / \mathrm{kg})$, acepromazine $(0.1 \mathrm{mg} / \mathrm{kg})$, glycopyrrolate $(0.01 \mathrm{mg} / \mathrm{kg})$, and ketamine/diazepam $(0.11 \mathrm{ml} / \mathrm{kg}$ in a $1: 1$ ratio). Cats were shaved and then cleaned using chlorhexidine soap. Gaseous anesthesia (isoflurane $1.5 \%-3 \%$ ) was initiated by mask followed by intubation. Isoflurane concentration was adjusted by monitoring cardiac/ respiratory rates and limb withdrawal with pressure applied to the paw. Body temperature was monitored with a rectal thermometer during surgery and maintained between $35^{\circ} \mathrm{C}$ and $37^{\circ} \mathrm{C}$ with a water-filled heating pad placed under the animal and an infrared lamp $\sim 50 \mathrm{~cm}$ over it.

For EMG, pairs of Teflon-insulated multistrain fine wires (AS633; Cooner Wire) were directed subcutaneously from a head-mounted 34pin connector (Omnetics) and sewn into the belly of selected forelimb and hindlimb muscles for bipolar recordings. Electrode placement was verified during surgery by electrically stimulating each muscle through the appropriate head connector channel. For bipolar nerve stimulation, pairs of Teflon-insulated multistrain fine wires (AS633; Cooner Wire) were directed subcutaneously from a head-mounted 4-pin connector (Omnetics). Wires were fixed inside silicon tubes $(\sim 1 \mathrm{~cm})$ that were placed around the right and left SP nerves at the ankle level and the right and left SR nerves at wrist level.

At the end of surgery, an antibiotic (Convenia, $0.1 \mathrm{ml} / \mathrm{kg}$ ) was injected subcutaneously and a transdermal fentanyl patch $(25 \mathrm{mcg} / \mathrm{h})$ was taped to the back of the animal $2-3 \mathrm{~cm}$ rostral to the base of the tail. Buprenorphine $(0.01 \mathrm{mg} / \mathrm{kg})$, a fast-acting analgesic, was also injected subcutaneously at the end of the surgery and $\sim 7 \mathrm{~h}$ later. Cats were then placed in an incubator and closely monitored until they regained consciousness. Five to $7 \mathrm{~d}$ after surgery, the fentanyl patch was removed. At the conclusion of the experiments, cats received a lethal dose of pentobarbital through the left or right cephalic vein.

Experimental design. Cats were initially trained to step on an animal treadmill (Bertec) for several consecutive minutes using food and affection as reward. During experiments, individual nerves were electrically stimulated (Grass S88 stimulator) with a train of three $0.2 \mathrm{~ms}$ pulses at $300 \mathrm{~Hz}$. Simulation intensity was set at 1.2 times the motor threshold (the minimal intensity required to elicit a small motor response in a homonymous flexor muscle). Two different nerve stimulations, or trials, were generally done in a single session, with each trial consisting of 5-7 min of consecutive locomotion. During a trial, $\sim 120$ stimulations were applied pseudo-randomly every $2-4$ locomotor cycles at varying delays following a hindlimb extensor burst onset to evoke responses at different times during the step cycle. The motor threshold was verified at the end of each session.

Reflex analysis. The methodology for quantifying reflexes is described in previous papers (Frigon and Rossignol, 2007, 2008b; Frigon et al., 2009; Hurteau et al., 2017). Briefly, onsets and offsets of EMG bursts of an extensor muscle on the stimulated side (lateral gastrocnemius [LG] or vastus lateralis $[\mathrm{VL}]$ ) were manually tagged. The step cycle was then synchronized to the hindlimb extensor burst onset, and each cycle was 
classified as stimulated or control (nonstimulated) for both SP and SR stimulation trials. Cycles were then divided into 10 bins or subphases of equal duration. In cycles with a stimulation, the onset of the stimulus was used as the reference for the start of the cycle. Responses in each bin were averaged. Nonstimulated cycles were also divided into 10 bins and averaged to provide a baseline locomotor EMG (blEMG). The blEMG was superimposed on evoked responses in each bin. To quantify reflex responses, a window was set at the onset and offset of the evoked responses, defined as prominent negative or positive deflections away from the blEMG, using predefined latencies as guidelines (Duysens and Stein, 1978; Abraham et al., 1985; Pratt et al., 1991; Loeb, 1993) and 95\% confidence intervals. Using the terminology introduced by Duysens and Loeb (1980), short-latency $(\sim 8-10 \mathrm{~ms})$ excitatory and inhibitory responses are termed $\mathrm{P} 1$ and $\mathrm{N} 1$ responses, respectively. Responses in the crossed, homolateral, and diagonal limbs that had an onset $<18 \mathrm{~ms}$ were also classified as $\mathrm{P} 1$, as the minimal latencies for spino-bulbo-spinal reflexes in the cat are $\sim 18 \mathrm{~ms}$ (Shimamura and Livingston, 1963). Midlatency $(20-25 \mathrm{~ms})$ excitatory and inhibitory responses are termed P2 and N2 responses, respectively. Longer-latency ( $>35 \mathrm{~ms}$ ) excitatory responses are termed P3 responses. The EMG of reflex responses (from onset to offset) was then rectified, integrated, and subtracted from the blEMG to provide a net value. This net value was then divided by the blEMG to provide a normalized measure of reflex amplitude. The division is required to determine whether changes in reflex responses across the cycle are independent of changes in the level of blEMG activity (Matthews, 1986; Duysens et al., 1993; Frigon and Rossignol, 2008a; Frigon et al., 2009; Hurteau et al., 2017). To illustrate the phase-dependent modulation of reflexes during the step cycles, reflex responses for each muscle were normalized to the maximal value occurring in 1 of the 10 subphases.

Data acquisition and analysis. Videos of the left and right sides were captured with two cameras (Basler AcA640-100 gm) at 60 frames per second with a spatial resolution of $640 \times 480$ pixels. A custom-made Labview program acquired images and synchronized the cameras with the EMG. Videos were analyzed off-line at 60 frames per second using custom-made software. Paw contact, defined as the first frame where the paw made visible contact with the treadmill surface, and liftoff, defined as the most caudal displacement of the limb, were determined for the four limbs by visual inspection. Cycle duration was measured from successive paw contacts, whereas stance duration corresponded to the interval of time from paw contact to liftoff. Swing duration was measured as cycle duration minus stance duration. The temporal phase interval was measured for each pair of limbs by dividing the duration between the stance onsets of two limbs by the cycle duration of the first limb used in the calculation.

To determine whether nerve stimulation affected the locomotor pattern, we determined the onsets and offsets of EMG bursts of the two VL muscles, a knee extensor and indicator of hindlimb stance phase duration, and the two triceps brachii (Tri) muscles, an elbow extensor and indicator of forelimb stance phase duration, in cycles without (control bursts) and with stimulation of the SP or SR nerves. For quantitative analysis, we retained cycles where the stimulation occurred during midstance and mid-swing of the homonymous limb. Burst duration was measured as the interval of time from burst onset to offset. Stimulated bursts were expressed as a percentage difference from control bursts (mean $\pm \mathrm{SD})$.

Statistical analysis. Reflex responses were quantified in the semitendinosus (St), anterior sartorius (Srt), VL, LG, biceps brachii (BB), Tri, and extensor carpi ulnaris (ECU). In the 5 cats, both the left and right SP nerves evoked responses $(n=10$ trials with SP nerve stimulation), whereas in 2 of 4 cats both the left and right SR nerves evoked responses ( $n=6$ trials with SR nerve stimulation). As such, responses evoked by SP and SR nerve stimulations were treated as separate cases for statistical analysis. The number of nerves and cats included in the analysis for each muscle is indicated in the figure legends. To determine whether homonymous, crossed, homolateral, and diagonal reflex responses were modulated with phase, we performed a one-factor (phase) repeated-measures ANOVA for each type of response (P1, P2, P3, N1, N2) on pooled data followed by pairwise comparisons. To determine the type of response that was strongest and had a higher probability of being evoked, we performed a one-factor (type of response) ANOVA on pooled data for SR and SP nerve stimulations followed by Tukey's test. We also performed a one-factor (type of response) ANOVA on the latencies of responses evoked by SR and SP nerve stimulations for pooled data followed by Tukey's test. To determine whether SP and SR nerve stimulation affected the locomotor pattern, a one-factor (control vs stim) ANOVA was performed on extensor burst durations of the four limbs as well as on the onset of the homonymous extensor burst for pooled data followed by Tukey's test. An $\alpha$ error of $p<0.05$ was considered significant for all statistical tests.

\section{Results}

\section{Type of gait analyzed}

The goal of the present study was to characterize cutaneous reflexes in several forelimb and hindlimb muscles evoked by cutaneous inputs from all four limbs. However, before describing cutaneous reflexes, it is important to characterize the type of gait analyzed. Reflexes were investigated during treadmill locomotion at $0.4 \mathrm{~m} / \mathrm{s}$, which is in the range of preferred speeds $(0.4-0.6 \mathrm{~m} / \mathrm{s})$ for cats stepping on a treadmill (Frigon, 2011). The EMG was recorded from several forelimb and hindlimb muscles, and the onsets and offsets of the stance phases of the four limbs were measured from kinematic data (Fig. 1A). For the group, mean cycle, stance, and swing durations were $1.08 \pm 0.15 \mathrm{~s}, 0.80 \pm$ $0.11 \mathrm{~s}$, and $0.28 \pm 0.06 \mathrm{~s}$, respectively (data not shown). Cats maintained a lateral sequence where contact of the right hindlimb (RH) was sequentially followed by contact of the right forelimb (RF), the left hindlimb (LH) and the left forelimb (LF) (Fig. 1B). Homologous pairings of the forelimbs (RF-LF) and hindlimbs (RH-LH) showed a strict out-of-phase alternation (values $\sim 0.5$ ), whereas homolateral (RF-RH and LF-LH) and diagonal (RF-LH and LF-RH) pairings had phase values $\sim 0.8$ and $\sim 0.3$, respectively (Fig. 1C). Therefore, from the duty factors and limb pairings, cats used a walking type of gait, as defined previously (Hildebrand, 1976, 1989).

\section{Cutaneous reflex responses in hindlimb muscles}

In the present study, we characterized responses in different hindlimb muscles evoked by cutaneous inputs from all four limbs. In this and the next section, all figures (see Figs. 2-8) are organized in the same way: in each figure, panels $A$ and $B$, respectively, show responses evoked by inputs from the homonymous and contralateral homologous (crossed) limbs, whereas panels $C$ and $D$, respectively, show responses evoked by inputs from the homolateral and diagonal limbs. Each panel represents averaged waveforms in the 10 bins of the cycle with a window of $80 \mathrm{~ms}$ that starts $5 \mathrm{~ms}$ before the stimulation for a single cat. Black traces represent an average of the cycles that received a stimulation, and the gray traces represent the cycles with no stimulation (the blEMG). This superimposition illustrates how the stimulation produces reflex responses in the EMG pattern that deviate from the normal pattern of EMG. It is important to note that the vertical scale bars on the right of the waveforms, in arbitrary units (a.u.), differ between bins. Although this obscures some of the phase-dependent modulation, it highlights the pattern of evoked responses. If responses were normalized to the largest response occurring in one bin, some responses in other bins would not be easily visible. Aligned vertically to the right of the waveforms is the average rectified EMG trace of the muscle over the cycle normalized to the onset of a hindlimb extensor (VL or LG) on the side of the stimulation. The scatter plots below the waveforms show normalized reflex amplitudes (see Materials and Methods). Short-latency (P1/N1), mid-latency (P2/N2), and longer-latency (P3) responses are shown separately. 
A

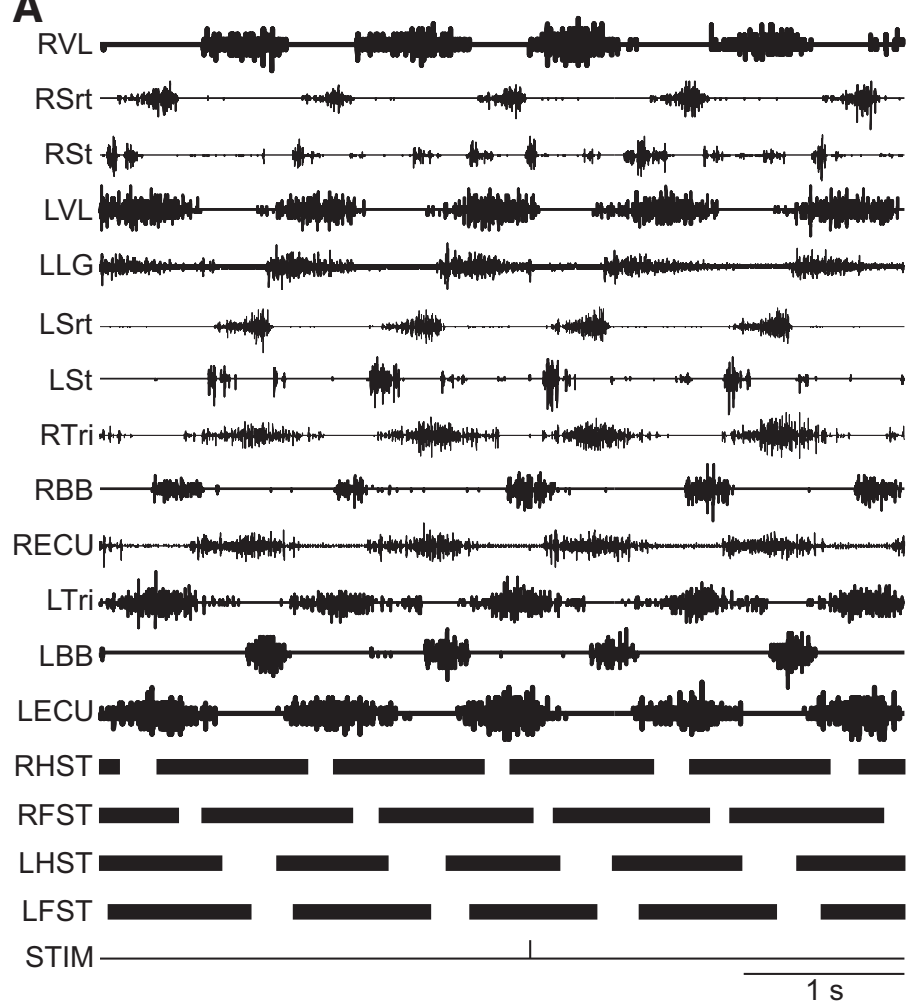

B

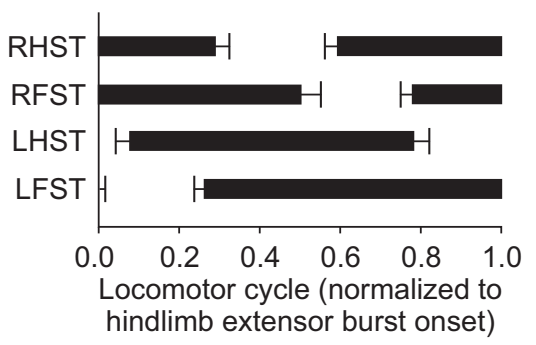

C

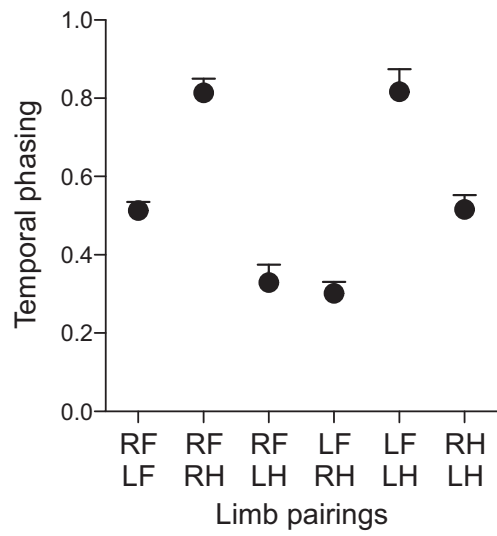

Figure 1. Type of gait analyzed. $\boldsymbol{A}$, Raw EMG waveforms from left (L) and right (R) limb muscles along with the stance phases (ST, thick black bars) of the four limbs during a $5 \mathrm{~s}$ period in 1 cat. In this episode, the left SP nerve was stimulated (STIM). B, Phase onsets and offsets were averaged from 16 separate locomotor trials, consisting of 10 SP nerve stimulations in 5 cats and six SR nerve stimulations in 4 cats. Error bars indicate mean \pm SD of the 16 trials. $C$, The temporal phase relationships between the six pairs of limbs were measured from the 16 trials and averaged. Data points are the mean \pm SD.

The St is a knee flexor and hip extensor muscle that has a brief burst around hindlimb swing onset and sometimes a second burst during early hindlimb stance (Fig. 1). Stimulating the homonymous SP nerve evoked $\mathrm{P} 1$ and $\mathrm{P} 2$ responses that peaked in amplitude when the muscle was active during late stance/early swing (Fig. 2A). These responses were qualitatively similar to previous studies in intact cats (Pratt et al., 1991; Bretzner and Drew, 2005; Frigon et al., 2009). Both P1 and P2 responses in the St muscle evoked by homonymous SP nerve stimulation were significantly modulated by phase for pooled data $(p=0.007$ and $p<0.0001$, respectively, ANOVA). Stimulating the SP nerve evoked $\mathrm{P} 2$ responses in the contralateral St, or crossed responses, that were larger in amplitude when the muscle was inactive (Fig. $2 B)$. These crossed P2 responses in St were significantly modulated by phase for pooled data ( $p=0.027$, ANOVA) and were qualitatively similar to a previous study (Frigon et al., 2009).

We found that responses in St evoked by inputs from the homolateral SR nerve were more complex than those from the diagonal SR nerve. In the example shown, P1 responses were followed by $\mathrm{P} 2$ responses when the muscle was inactive or by $\mathrm{N} 2$ responses when the muscle was active (Fig. 2C). Homolateral P1 and $\mathrm{N} 2 / \mathrm{P} 2$ responses in St were not significantly modulated by phase for pooled data ( $p=0.173$ and $p=0.222$, respectively, ANOVA). Responses evoked by inputs from the diagonal SR nerve consisted of $\mathrm{P} 1$ responses that were larger during stance (Fig. 2D). These diagonal $\mathrm{P} 1$ responses in St were not significantly modulated by phase for pooled data ( $p=0.099$, ANOVA). Thus, overall, responses in St evoked by inputs from the same side (homonymous, homolateral) were more complex, consisting of
$\mathrm{P} 1$ and $\mathrm{P} 2 / \mathrm{N} 2$, whereas those evoked by inputs from the other side (crossed, diagonal) consisted only of $\mathrm{P} 1$ responses.

The anterior portion of the Srt muscle is a hip flexor and knee extensor with a burst that covers most of the hindlimb swing phase (see Fig. 1). Stimulating the homonymous SP nerve evoked $\mathrm{N} 1$ responses that were followed by $\mathrm{P} 2$ responses when the muscle was active or mainly $\mathrm{P} 2$ responses when the muscle was inactive (Fig. 3A). These were qualitatively similar to previous studies in intact cats with SP nerve stimulation (Bretzner and Drew, 2005; Frigon et al., 2009). Both N1 and P2 responses in the Srt muscle evoked by homonymous SP nerve stimulation were significantly modulated by phase for pooled data $(p<0.0001$ and $p=0.0002$, ANOVA). Stimulating the SP nerve also evoked N1 and $\mathrm{P} 2$ responses in the contralateral Srt when the muscle was active (Fig. 3B), as described previously (Frigon et al., 2009). These crossed N1 and P2 responses in Srt were significantly modulated by phase for pooled data $(p=0.036$ and $p=0.024$, respectively, ANOVA).

Responses in Srt evoked by stimulating the homolateral SR nerve were described by Miller et al. (1977) in decerebrate cats during treadmill locomotion. They showed responses in Srt only during its period of activity, with inhibitory responses when Srt and quadriceps activities overlapped and excitatory responses during the rest of the period of Srt activity. Here, we extend the findings of Miller et al. (1977) by showing responses in the anterior Srt evoked by inputs from both homolateral and diagonal SR nerves. Stimulating the SR nerve evoked a similar pattern of responses in the homolateral (Fig. 3C) and diagonal (Fig. 3D) Srt that consisted of $\mathrm{P} 1, \mathrm{~N} 2$, and $\mathrm{P} 3$ responses when the muscle was 


\section{A St - homonymous}
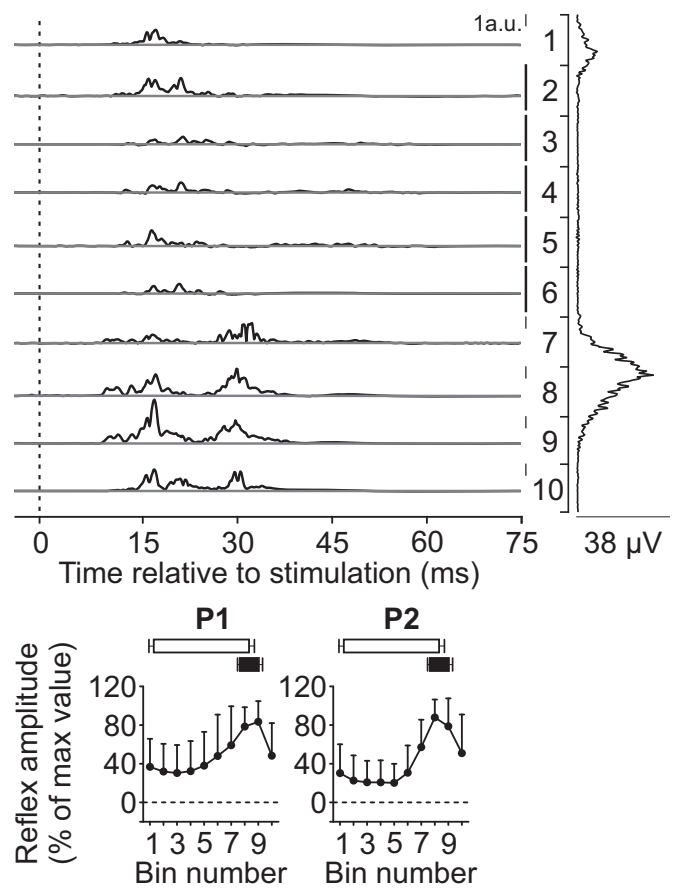

\section{St - homolateral}
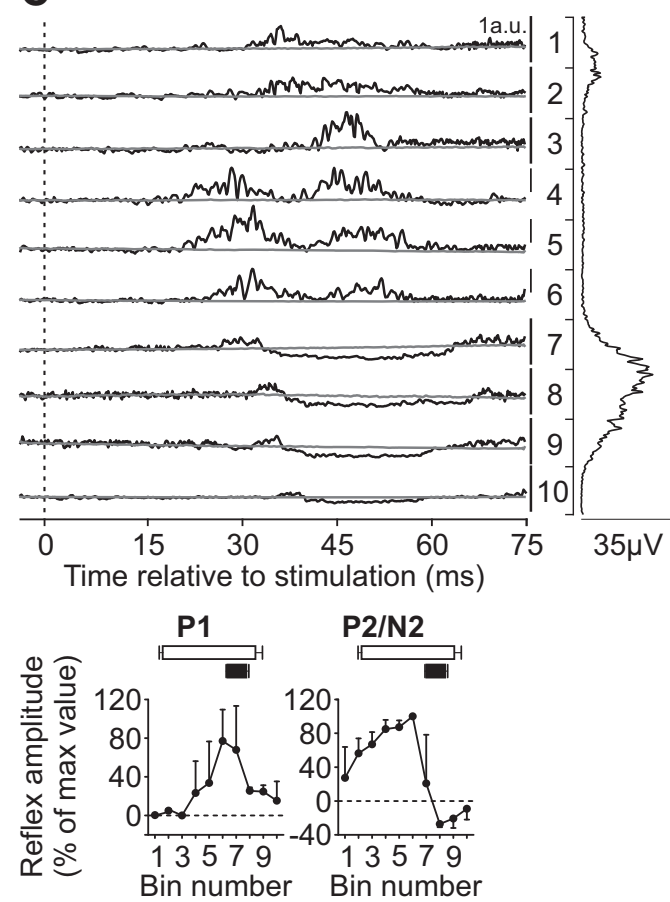

B St - crossed
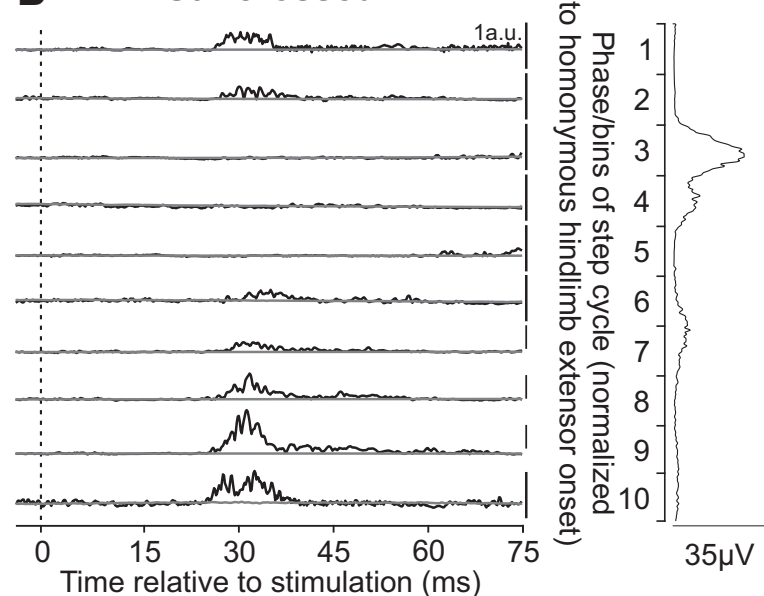

P2

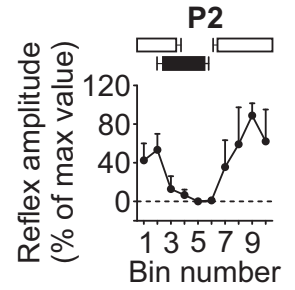

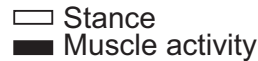
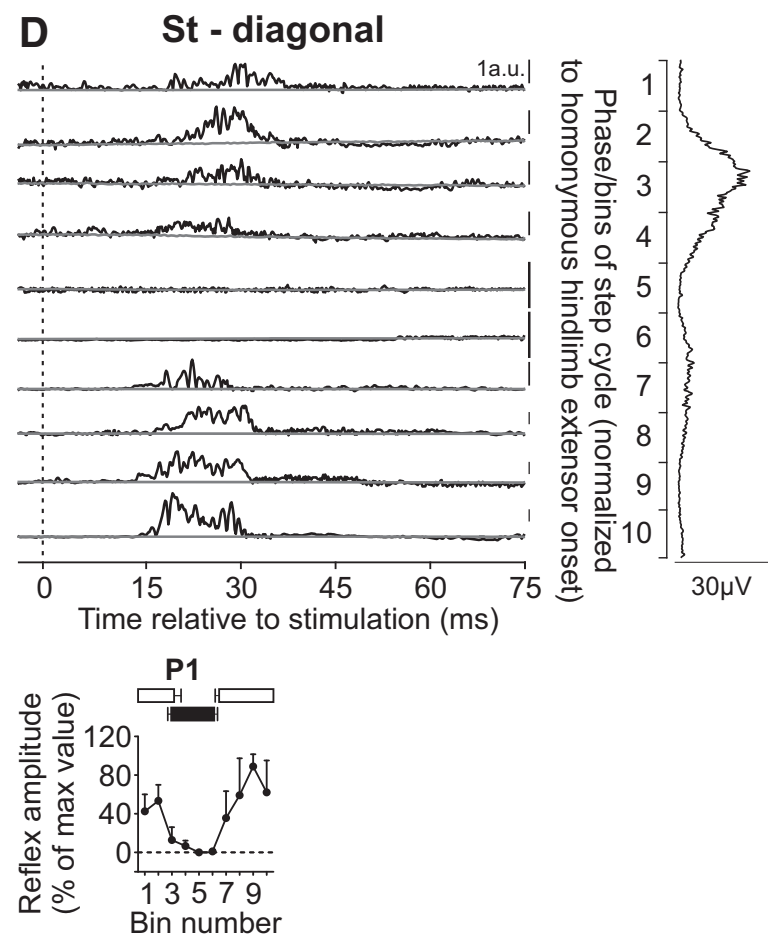

Figure 2. Intralimb and interlimb cutaneous reflexes in St. SP nerve stimulations evoked ( $\boldsymbol{A}$ ) homonymous ( 10 trials in 5 cats) and (B) crossed responses ( 3 trials in 2 cats), whereas SR nerve stimulations evoked $(\boldsymbol{C})$ homolateral ( 4 trials in 3 cats) and $(\boldsymbol{D})$ diagonal ( 3 trials in 2 cats) responses in the St. Each panel represents averaged waveforms in the 10 bins of the cycle with a window of $80 \mathrm{~ms}$ that starts $5 \mathrm{~ms}$ before stimulation in a single cat. Black traces represent averaged cycles that received a stimulation (i.e., reflex responses, $n=7-18$ stimuli per bin). Gray traces represent averaged cycles with no stimuli ( $n=83-146$ cycles). Scale bars are shown in arbitrary units (a.u.) for each bin and can differ to optimize evoked responses. Aligned vertically is the average rectified EMG trace of the St muscle over the cycle normalized to a hindlimb extensor on the side of the stimulation. Scatter plots represent reflex amplitudes (mean \pm SD) in each bin of the cycle expressed as a percentage of the maximal response found in one of the bins for pooled data. Short-latency (P1) and mid-latency (P2/N2) responses are shown separately.

active. The homolateral $\mathrm{P} 1$ and N2 responses in Srt were significantly modulated by phase for pooled data $(p=0.005$ and $p=$ 0.015 , respectively, ANOVA), whereas $\mathrm{P} 3$ responses were not significantly modulated ( $p=0.059$, ANOVA). Only N2 responses in the diagonal Srt were significantly modulated by phase for pooled data $(p=0.009)$, whereas $\mathrm{P} 1$ and $\mathrm{P} 3$ where not $(p=0.119$ and $p=0.128$, respectively, ANOVA). Thus, overall, responses in Srt evoked by inputs from homologous limbs showed a similar pattern of responses: N1/P2 from hindlimb inputs and P1/N2/P3 responses from forelimb inputs. 


\section{A Srt-homonymous}
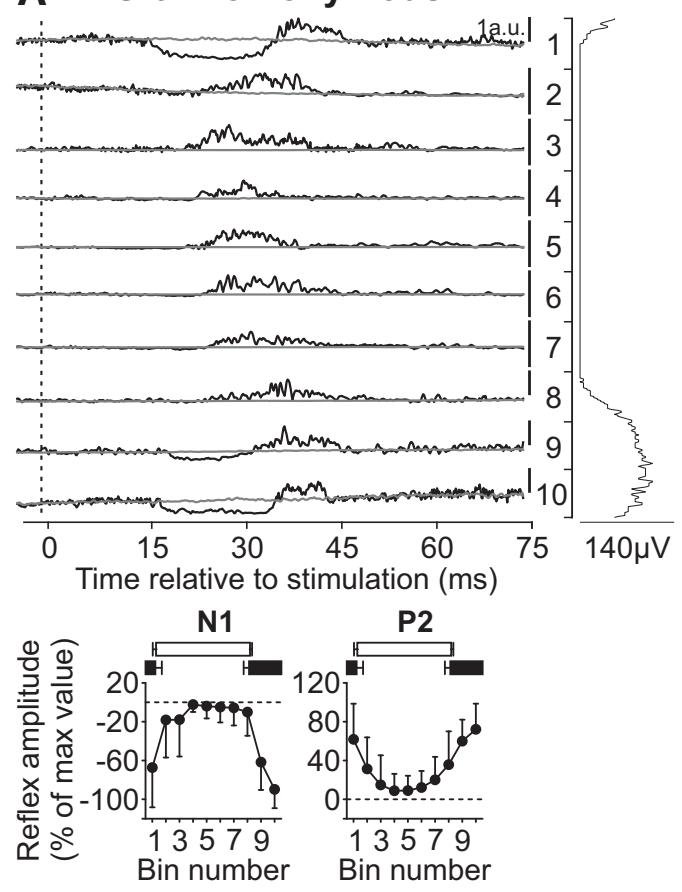

\section{Srt - homolateral}
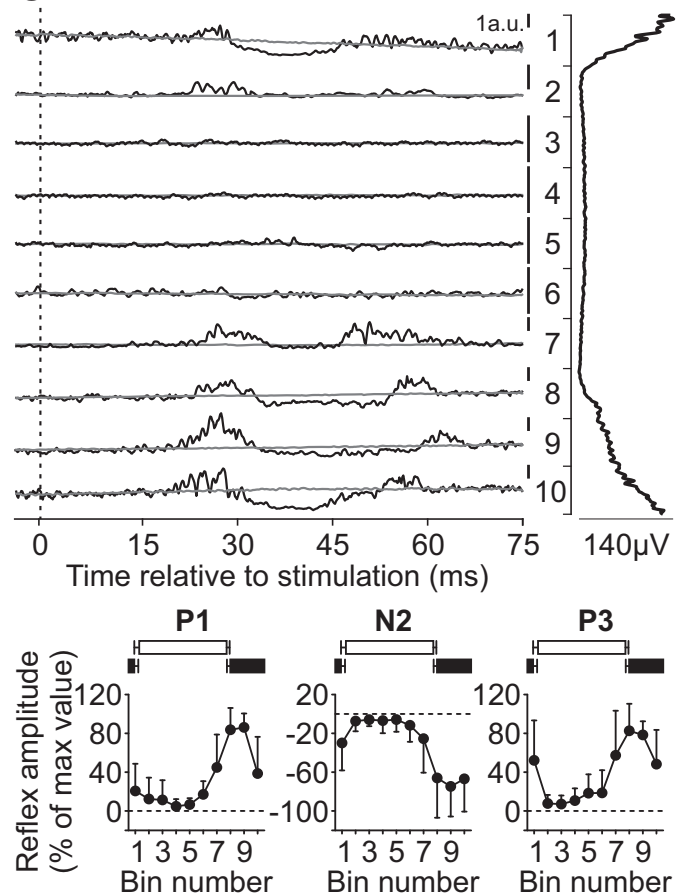
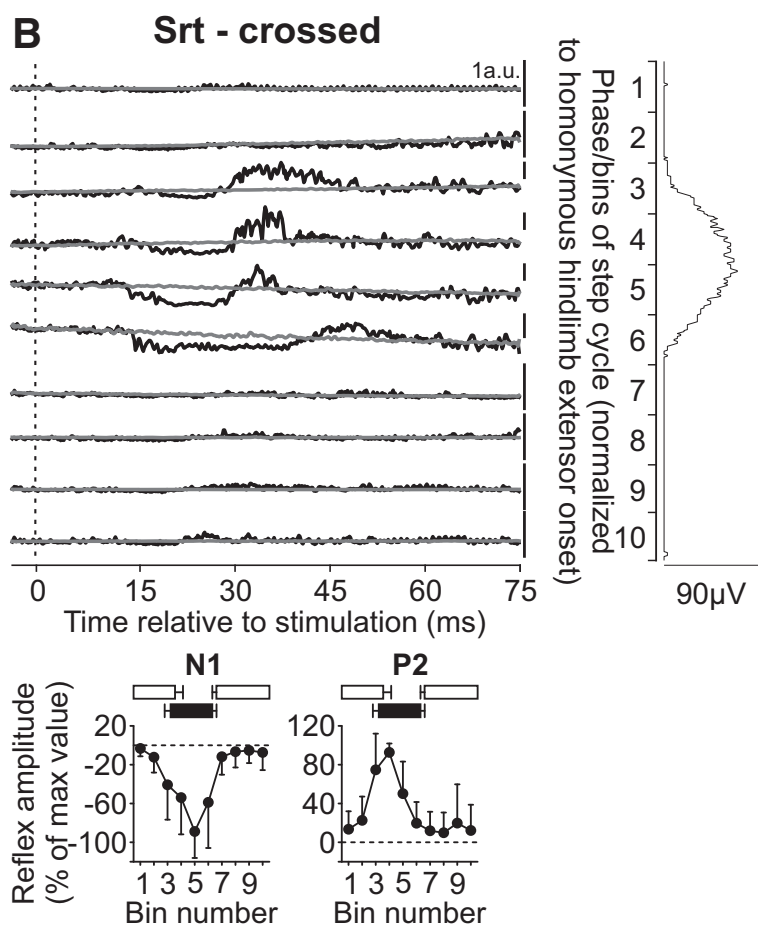

$\square$ Stance

Muscle activity

\section{Srt - diagonal}
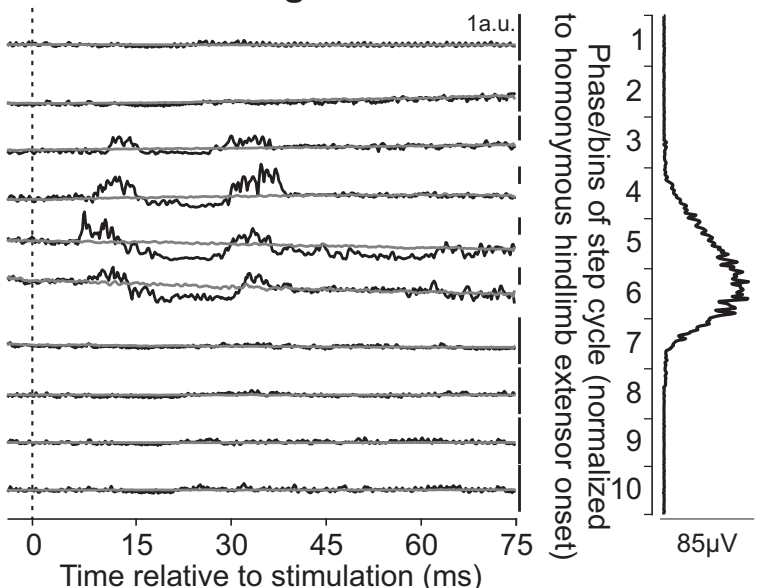

P1
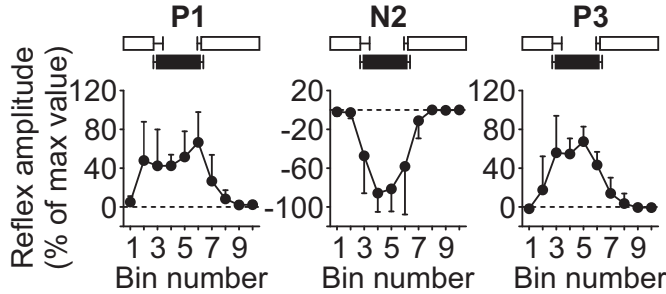

Figure 3. Intralimb and interlimb cutaneous reflexes in anterior Srt. SP nerve stimulations evoked ( $\boldsymbol{A}$ ) homonymous (10 trials in 5 cats) and ( $\boldsymbol{B})$ crossed responses ( 6 trials in 4 cats), whereas SR nerve stimulations evoked $(\boldsymbol{C})$ homolateral ( 3 trials in 3 cats) and $(\boldsymbol{D})$ diagonal ( 5 trials in 3 cats) responses in the Srt. Each panel represents averaged waveforms in the 10 bins of the cycle with a window of 80 ms that starts 5 ms before stimulation in a single cat. Black traces represent averaged cycles that received a stimulation (i.e., reflex responses, $n=7-18$ stimuli per bin). Gray traces represent averaged cycles with no stimuli ( $n=83-146$ cycles). Scale bars are shown in arbitrary units (a.u.) for each bin and can differ to optimize evoked responses. Aligned vertically is the average rectified EMG trace of the Srt muscle over the cycle normalized to a hindlimb extensor on the side of the stimulation. Scatter plots represent reflex amplitudes (mean \pm SD) in each bin of the cycle expressed as a percentage of the maximal response found in one of the bins for pooled data. Short-latency (P1/N1), mid-latency (P2/N2), and longer-latency (P3) responses are shown separately.

The VL muscle is a knee extensor with a burst that covers most of the hindlimb stance phase (Fig. 1). Stimulating the homonymous SP nerve evoked N1 responses that were followed by P2 responses when the muscle was active or $\mathrm{P} 1 / \mathrm{P} 2$ responses toward the end of the burst and when the muscle was inactive (Fig. 4A). The N1/P1 and P2 responses in the VL muscle evoked by homonymous SP nerve stimulation were significantly modulated by phase for pooled data (both $p<0.0001$, ANOVA). Stimulating 


\section{A VL - homonymous}
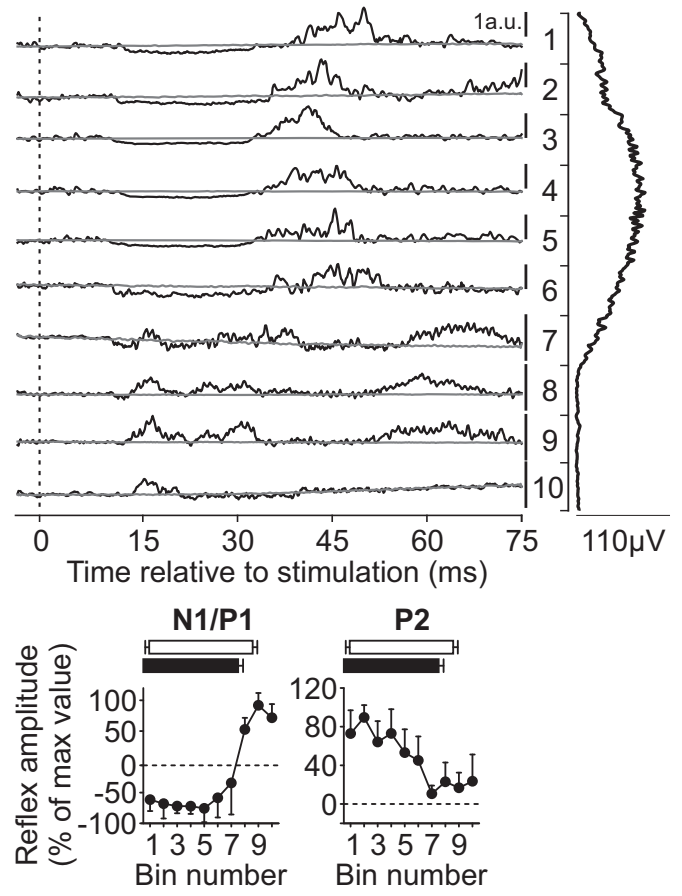

C VL - homolateral
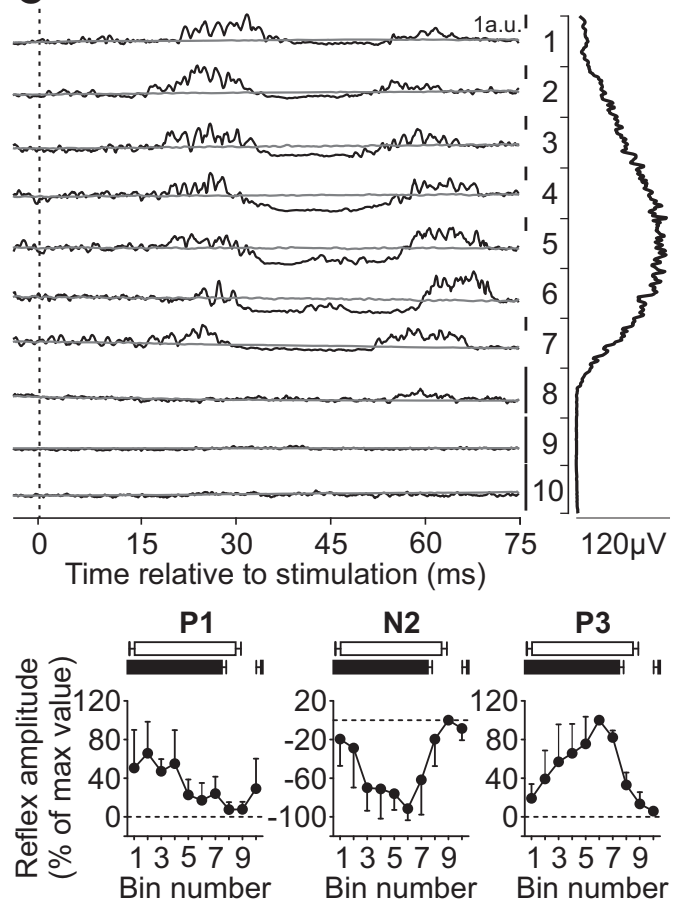

B VL - crossed
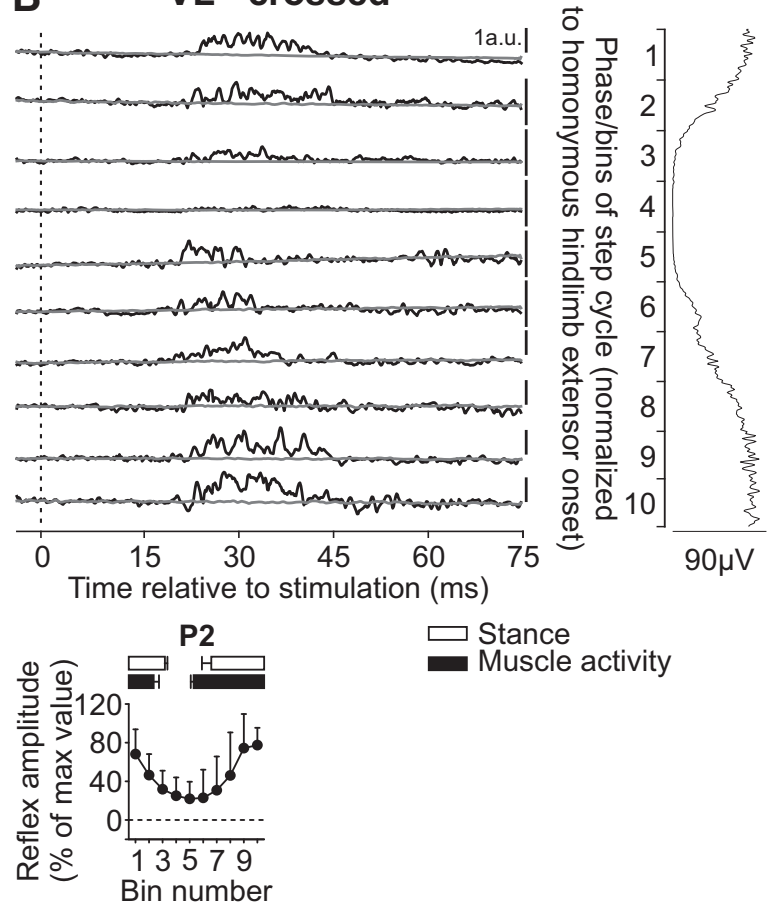

$\square$ Stance

Muscle activity
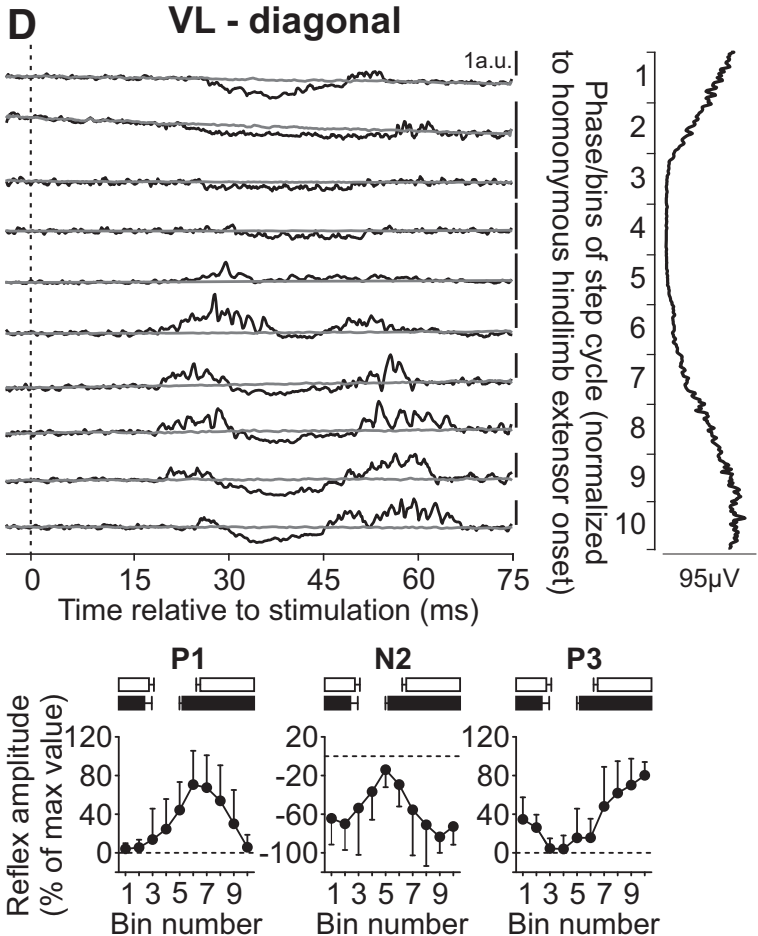

Figure 4. Intralimb and interlimb cutaneous reflexes in VL. SP nerve stimulations evoked ( $\boldsymbol{A})$ homonymous ( 6 trials in 5 cats) and (B) crossed responses ( 6 trials in 5 cats), whereas SR nerve stimulations evoked ( $($ homolateral ( 3 trials in 2 cats) and (D) diagonal ( 5 trials in 3 cats) responses in the VL. Each panel represents averaged waveforms in the 10 bins of the cycle with a window of $80 \mathrm{~ms}$ that starts $5 \mathrm{~ms}$ before stimulation in a single cat. Black traces represent averaged cycles that received a stimulation (i.e., reflex responses, $n=7-16$ stimuli per bin). Gray traces represent averaged cycles with no stimuli ( $n=83-146$ cycles). Scale bars are shown in arbitrary units (a.u.) for each bin and can differ to optimize evoked responses. Aligned vertically is the average rectified EMG trace of the VL muscle over the cycle normalized to a hindlimb extensor on the side of the stimulation. Scatter plots represent reflex amplitudes (mean \pm SD) in each bin of the cycle expressed as a percentage of the maximal response found in one of the bins for pooled data. Short-latency (P1/N1), mid-latency (P2/N2), and longer-latency (P3) responses are shown separately.

the SP nerve evoked P2 responses in the contralateral VL primarily when the muscle was active (Fig. $4 B$ ). These crossed P2 responses in VL were significantly modulated by phase for pooled data $(p=0.027$, ANOVA $)$.
Responses in the quadriceps evoked by stimulating the homolateral SR nerve were described by Miller et al. (1977) in decerebrate cats during treadmill locomotion. Responses were only present during the period of activity and were excitatory. How- 
ever, it is not clear which quadriceps muscle was recorded and their technique did not allow for the identification of inhibitory responses. Here, we extend the findings of Miller et al. (1977) by showing responses in the VL evoked by inputs from homolateral and diagonal SR nerves in intact cats. Stimulating the SR nerve evoked a similar pattern of responses in the homolateral (Fig. 4C) and diagonal (Fig. 4D) VL that consisted of P1, N2, and P3 responses primarily when the muscle was active. The homolateral $\mathrm{P} 1, \mathrm{~N} 2$, and $\mathrm{P} 3$ responses in VL were not significantly modulated by phase for pooled data ( $p=0.371, p=0.356$, and $p=0.274$, respectively, ANOVA). The diagonal P1 and P3 responses in VL were significantly modulated by phase for pooled data $(p=0.018$ and $p=0.039$, respectively, ANOVA), but $\mathrm{N} 2$ was not ( $p=$ 0.191 , ANOVA).

The LG muscle is an ankle extensor and knee flexor with a burst that covers most of the hindlimb stance phase (Fig. 1). Stimulating the homonymous SP nerve evoked $\mathrm{N} 1$ responses that were followed by $\mathrm{P} 2$ responses when the muscle was active or $\mathrm{P} 1 / \mathrm{P} 2$ responses toward the end of the burst and when the muscle was inactive (Fig. 5A). These were qualitatively similar to previous studies in intact cats with SP nerve stimulation (Abraham et al., 1985; Bretzner and Drew, 2005; Frigon and Rossignol, 2008b; Frigon et al., 2009). The N1/P1 and P2 responses in the LG muscle evoked by homonymous SP nerve stimulation were significantly modulated by phase for pooled data $(p=0.031$ and $p=0.028$, respectively, ANOVA). Stimulating the SP nerve evoked N1 responses that were followed by $\mathrm{P} 2$ responses in the contralateral LG primarily when the muscle was active (Fig. $5 B$ ), as described previously (Frigon and Rossignol, 2008b). The crossed P2, but not $\mathrm{N} 1$, responses in LG were significantly modulated by phase for pooled data ( $p=0.001$ and $p=0.074$, respectively, ANOVA).

Responses in the LG muscle evoked by stimulating forelimb nerves have not been described. Stimulating the SR nerve evoked a similar pattern of responses in the homolateral (Fig. $5 C$ ) and diagonal (Fig. 5D) LG that consisted of P1, N2, and P3 responses primarily when the muscle was active. The homolateral and diagonal P1, N2, and P3 responses in LG were not significantly modulated by phase for pooled data (homolateral: $p=0.359, p=$ 0.240 and $p=0.359$, respectively; diagonal: $p=0.233, p=0.337$, and $p=0.193$, respectively, ANOVA). Thus, responses in the homonymous, homolateral, and diagonal LG and VL muscles, both extensors that are active throughout stance, are similar. The main difference between these two extensors is the lack of N1 responses in the crossed VL, as noted by Frigon and Rossignol (2008a).

In summary, inputs from all four limbs evoked responses in a given hindlimb muscle, including muscles that flex (Srt) or extend (St) the hip, flex (St, LG) or extend (VL, Srt) the knee and extend (LG) the ankle. The pattern of evoked responses depended on the muscle and the input limb (the limb stimulated). The majority of reflex responses evoked in hindlimb muscles was significantly phase-dependent.

\section{Cutaneous reflex responses in forelimb muscles}

In contrast to hindlimb muscles, only a handful of studies have described cutaneous reflexes in forelimb muscles during real or fictive locomotion by stimulating forelimb nerves (Miller et al., 1977; Drew and Rossignol, 1985, 1987; Hishinuma and Yamaguchi, 1989; Fuwa et al., 1991; Seki and Yamaguchi, 1997). Surprisingly, crossed reflex responses between the forelimbs have not been described during real locomotion in the intact cat, and only one study in decerebrate cats has described homolateral re- sponses (Miller et al., 1977) with no mention of diagonal responses.

The long head of the BB muscle is primarily an elbow flexor but also a shoulder flexor with a burst that covers most of the forelimb swing phase (Fig. 1). Reflex responses in the BB muscle evoked by stimulating the homonymous SR nerve during cat locomotion have been described as short-latency excitatory responses when the muscle is active (Miller et al., 1977; Drew and Rossignol, 1987; Hishinuma and Yamaguchi, 1989; Fuwa et al., 1991; Seki and Yamaguchi, 1997). In the present study, stimulating the homonymous SR nerve evoked $\mathrm{P} 1$ and $\mathrm{P} 2$ responses (Fig. $6 A)$. The $\mathrm{P} 1$ responses peaked in amplitude when the $\mathrm{BB}$ muscle was active while $\mathrm{P} 2$ responses peaked toward the end of the burst or when the muscle was inactive. Both $\mathrm{P} 1$ and $\mathrm{P} 2$ responses in the $\mathrm{BB}$ muscle evoked by homonymous SR nerve stimulation were significantly modulated by phase for pooled data $(p=0.018$ and $p=0.014$, respectively, ANOVA). Stimulating the SR nerve primarily evoked P1 responses in the contralateral $\mathrm{BB}$, or crossed responses, that peaked in amplitude just before the burst started or in its early portion (Fig. $6 B$ ). The crossed $\mathrm{P} 1$ responses in $\mathrm{BB}$ were significantly modulated by phase for pooled data $(p=$ 0.003, ANOVA).

Responses in BB evoked by stimulating the homolateral SP nerve were described by Miller et al. (1977) in decerebrate cats during treadmill locomotion. Homolateral responses in $\mathrm{BB}$ were small but were phase-modulated with excitatory responses present just before and during its period of activity. Here, we extend the findings of Miller et al. (1977) by showing responses in the BB evoked by inputs from homolateral and diagonal SP nerves. Stimulating the SP nerve evoked $\mathrm{N} 1$ and $\mathrm{P} 2$ responses in the homolateral $\mathrm{BB}$ when the muscle was active (Fig. $6 \mathrm{C}$ ), whereas it evoked $\mathrm{P} 1$ and $\mathrm{N} 2$ responses in the diagonal $\mathrm{BB}$ when the muscle was active (Fig. 6D). The homolateral (N1/P2) and diagonal (P1/ $\mathrm{N} 2$ ) responses in BB were significantly modulated by phase for pooled data (homolateral: $p=0.001$ and $p=0.006$; diagonal: $p=$ 0.027 and $p=0.014$, ANOVA).

The long head of the Tri muscle is primarily an elbow extensor but also a shoulder extensor with a burst that covers most of the forelimb stance phase (Fig. 1). Reflex responses in the Tri muscle evoked by stimulating the homonymous SR nerve have been described during cat (Miller et al., 1977; Drew and Rossignol, 1985, 1987; Hishinuma and Yamaguchi, 1989; Seki and Yamaguchi, 1997) locomotion, consisting of P1 responses during swing and $\mathrm{N} 1$ followed by P2 responses during stance. In the present study, stimulating the homonymous SR nerve evoked $\mathrm{N} 1$ responses that were followed by $\mathrm{P} 2$ responses in Tri when the muscle was active (Fig. 7A). Both N1 and P2 responses in the Tri muscle evoked by homonymous $\mathrm{SR}$ nerve stimulation were significantly modulated by phase for pooled data ( $p=0.036$ and $p=0.002$, respectively, ANOVA). Stimulating the SR nerve primarily evoked P1 responses in the contralateral Tri that were followed by $\mathrm{N} 2$ and then P3 responses when the muscle was active or simply P1 responses when the muscle was inactive (Fig. $7 B$ ). Only the crossed N2 responses in Tri were significantly modulated by phase for pooled data $(p=0.047$, ANOVA), whereas $\mathrm{P} 1$ and $\mathrm{P} 3$ were not $(p=$ 0.245 and $p=0.298$, respectively, ANOVA).

Responses in Tri evoked by stimulating the homolateral SP nerve were described by Miller et al. (1977) in decerebrate cats during treadmill locomotion. Excitatory homolateral responses were observed around its onset of activity. Here, we extend the findings of Miller et al. (1977) by showing responses in Tri evoked by inputs from homolateral and diagonal SP nerves. Stimulating the SP nerve evoked P1 and N2 responses in the homolateral Tri 


\section{A LG - homonymous}
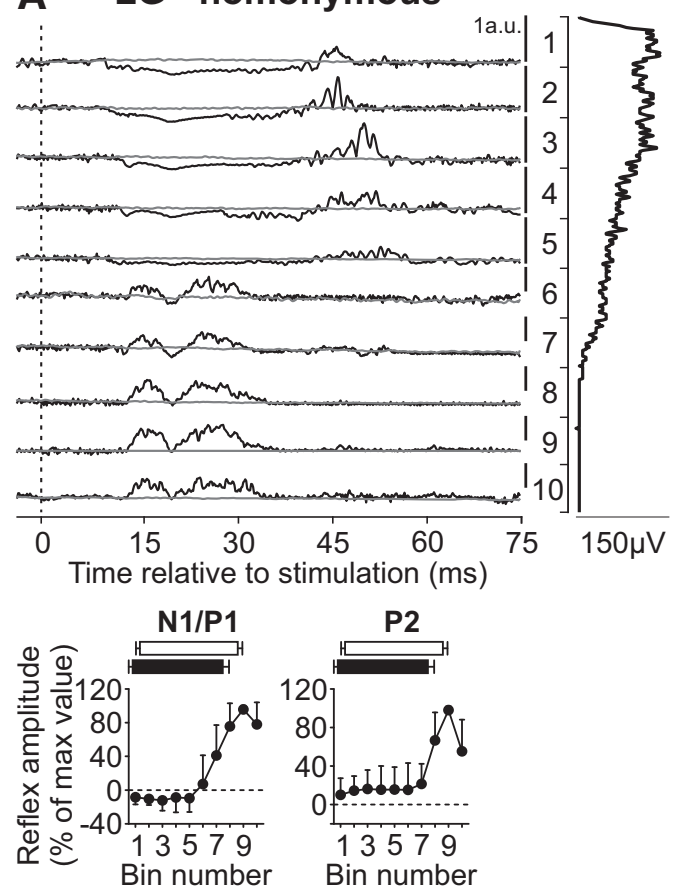

\section{LG - homolateral}
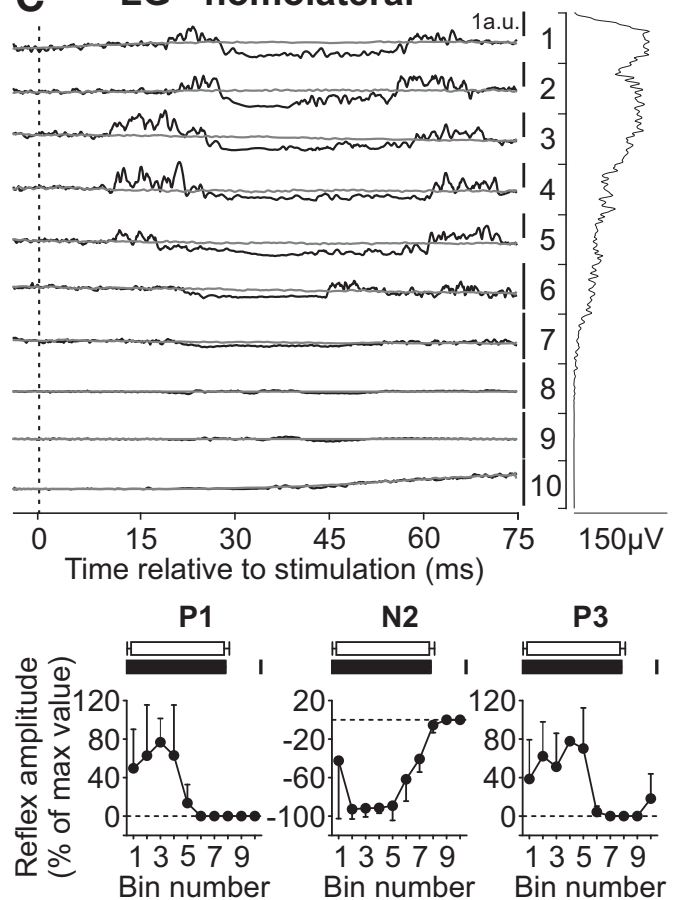

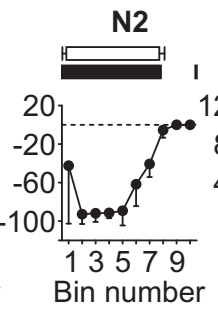

P3

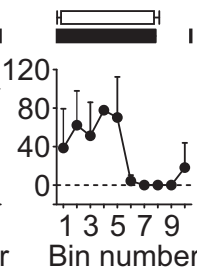

B LG - crossed
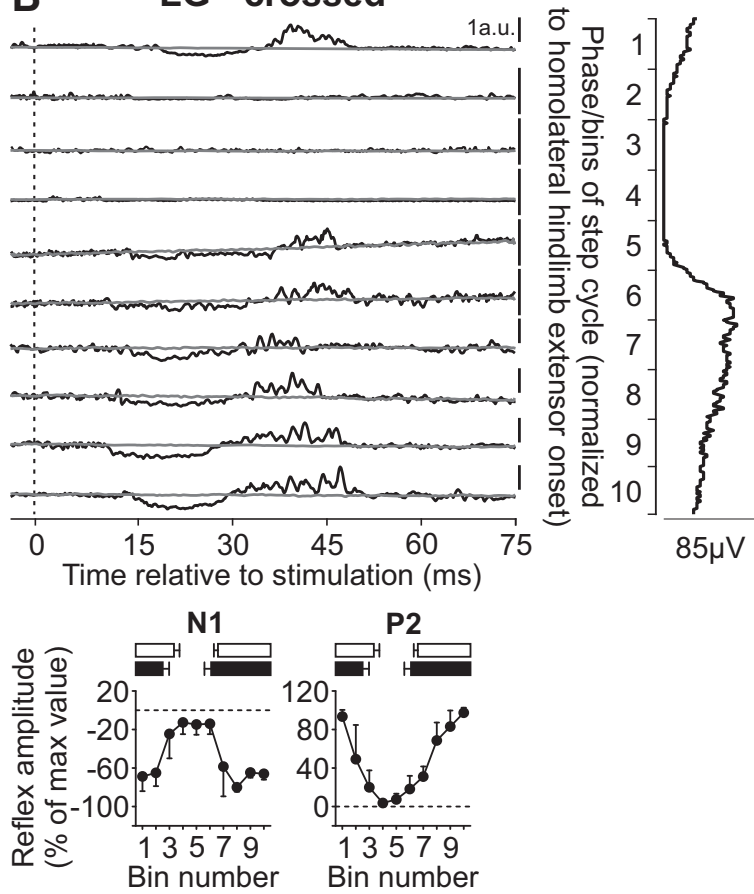

$\square$ Stance

Muscle activity
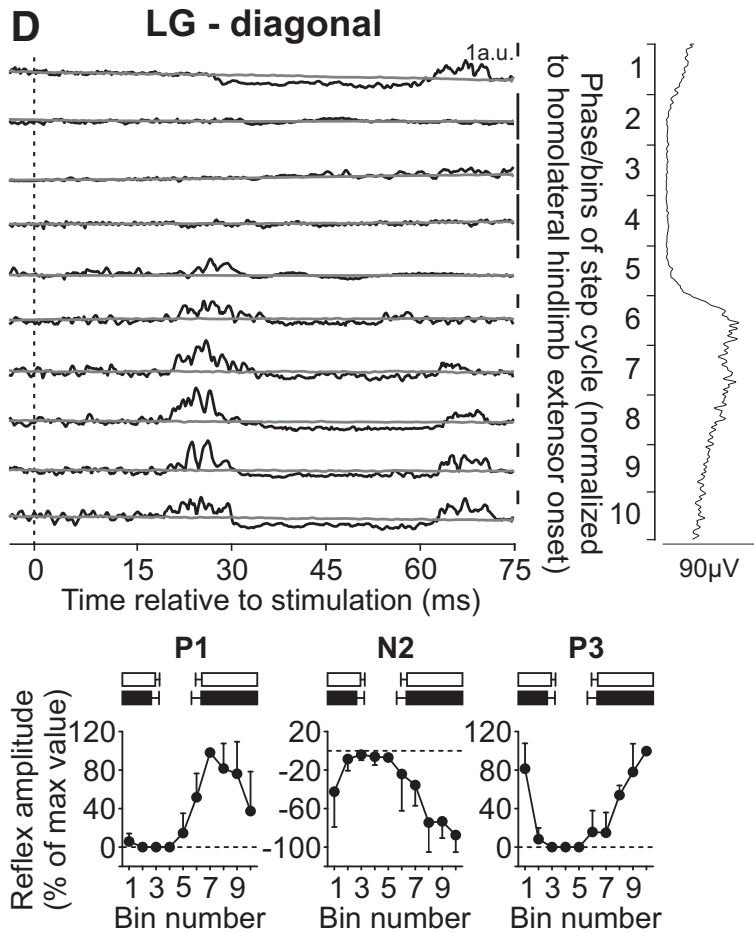

Figure 5. Intralimb and interlimb cutaneous reflexes in LG. SP nerve stimulations evoked ( $\boldsymbol{A}$ ) homonymous ( 4 trials in 3 cats) and ( $\boldsymbol{B})$ crossed responses ( 4 trials in 3 cats), whereas SR nerve stimulations evoked ( $\boldsymbol{C}$ homolateral ( 2 trials in 2 cats) and (D) diagonal ( 2 trials in 2 cats) responses in the LG. Each panel represents averaged waveforms in the 10 bins of the cycle with a window of 80 ms that starts $5 \mathrm{~ms}$ before stimulation in a single cat. Black traces represent averaged cycles that received a stimulation (i.e., reflex responses, $n=5-24$ stimuli per bin). Gray traces represent averaged cycles with no stimuli ( $n=83-126$ cycles). Scale bars are shown in arbitrary units (a.u.) for each bin and can differ to optimize evoked responses. Aligned vertically is the average rectified EMG trace of the LG muscle over the cycle normalized to a hindlimb extensor on the side of the stimulation. Scatter plots represent reflex amplitudes (mean \pm SD) in each bin of the cycle expressed as a percentage of the maximal response found in one of the bins for pooled data. Short-latency (P1/N1), mid-latency (P2/N2), and longer-latency (P3) responses are shown separately.

when the muscle was active (Fig. 7C), whereas it evoked only N1 responses in the diagonal Tri when the muscle was active (Fig. $7 D)$. Homolateral (P1 and $\mathrm{N} 2$ ) and diagonal (N1) responses in Tri were significantly modulated by phase for pooled data $(p=$ $0.003, p=0.004$, and $p=0.018$, respectively, ANOVA).
The ECU muscle is primarily a wrist extensor with a burst that covers most of the forelimb stance phase (Fig. 1). Reflex responses in the ECU muscle evoked by stimulating the homonymous SR nerve have been described during cat locomotion (Drew and Rossignol, 1987), consisting of P1 responses during 


\section{A BB - homonymous}

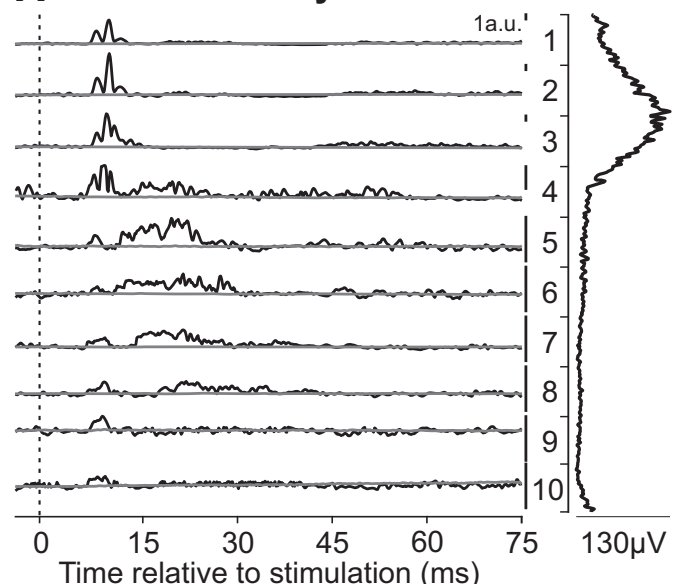

Time relative to stimulation (ms)

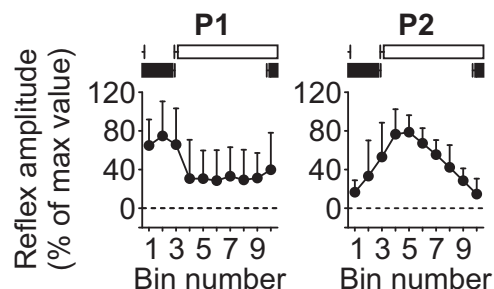

\section{BB - homolateral}
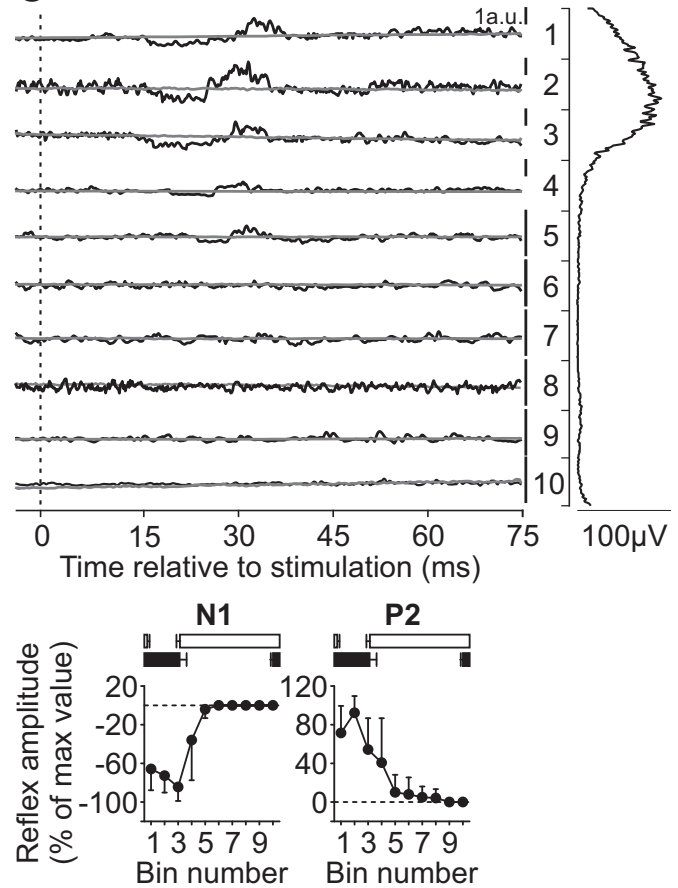

B BB - crossed
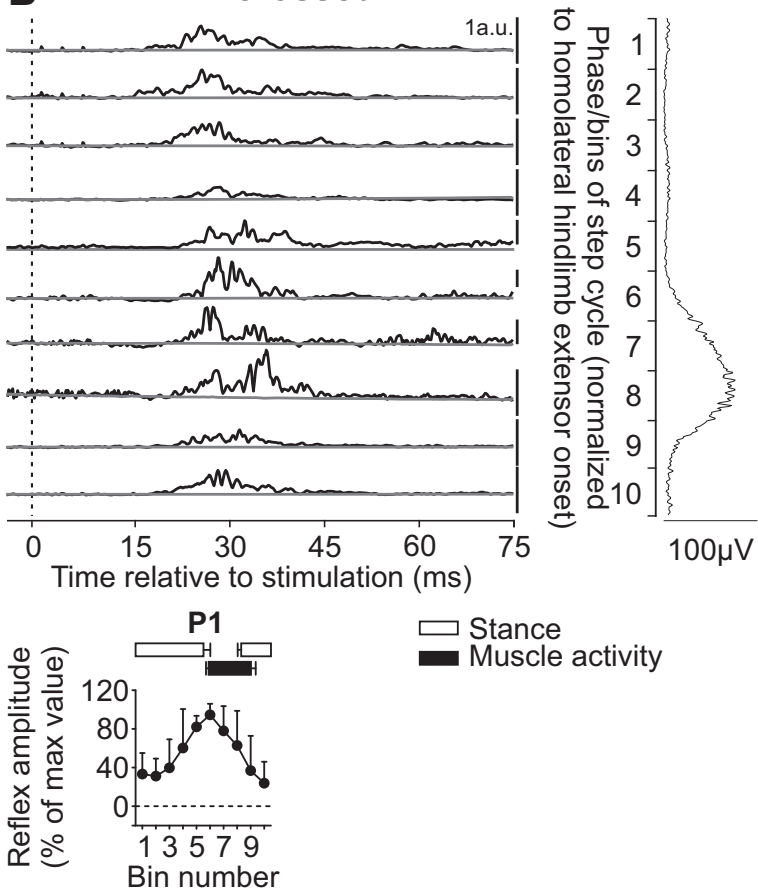

D BB - diagonal
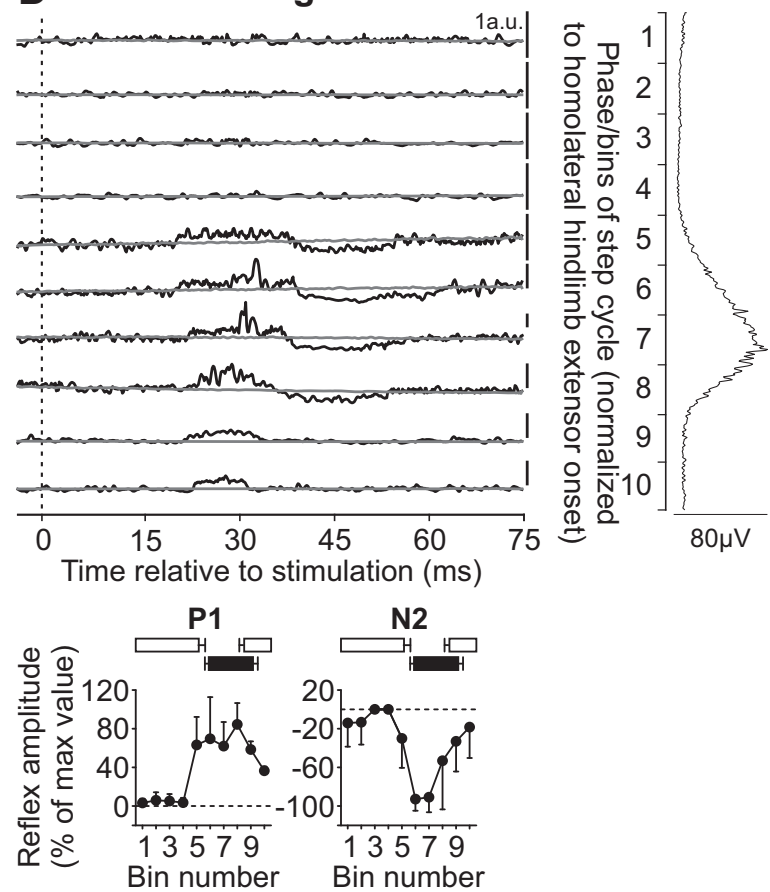

Figure 6. Intralimb and interlimb cutaneous reflexes in BB. SR nerve stimulations evoked ( $\boldsymbol{A}$ ) homonymous ( 6 trials in 4 cats) and ( $\boldsymbol{B}$ ) crossed responses ( 5 trials in 3 cats), whereas SP nerve stimulations evoked ( $C$ homolateral ( 5 trials in 3 cats) and $(\boldsymbol{D})$ diagonal ( 3 trials in 2 cats) responses in the BB. Each panel represents averaged waveforms in the 10 bins of the cycle with a window of 80 ms that starts 5 ms before stimulation in a single cat. Black traces represent averaged cycles that received a stimulation (i.e., reflex responses, $n=6-22$ stimuli per bin). Gray traces represent averaged cycles with no stimuli ( $n=83-146$ cycles). Scale bars are shown in arbitrary units (a.u.) for each bin and can differ to optimize evoked responses. Aligned vertically is the average rectified EMG trace of the BB muscle over the cycle normalized to a hindlimb extensor on the side of the stimulation. Scatter plots represent reflex amplitudes (mean \pm SD) in each bin of the cycle expressed as a percentage of the maximal response found in one of the bins for pooled data. Short-latency (P1/N1) and mid-latency (P2/N2) responses are shown separately.

swing and $\mathrm{N} 1$ followed by $\mathrm{P} 2$ responses during stance. In the present study, stimulating the homonymous SR nerve evoked $\mathrm{N} 1$ responses that were followed by $\mathrm{P} 2$ responses in ECU when the muscle was active (Fig. 8A). Both $\mathrm{N} 1$ and $\mathrm{P} 2$ responses in the ECU muscle evoked by homonymous SR nerve stimula- tion were significantly modulated by phase for pooled data ( $p=0.005$ and $p<0.0001$, respectively, ANOVA). Stimulating the SR nerve evoked $\mathrm{P} 1$ responses in the crossed ECU that were followed by $\mathrm{N} 2$ or $\mathrm{P} 2$ responses and then $\mathrm{P} 3$ responses when the muscle was active or simply $\mathrm{P} 2$ responses when the 


\section{A Tri - homonymous}
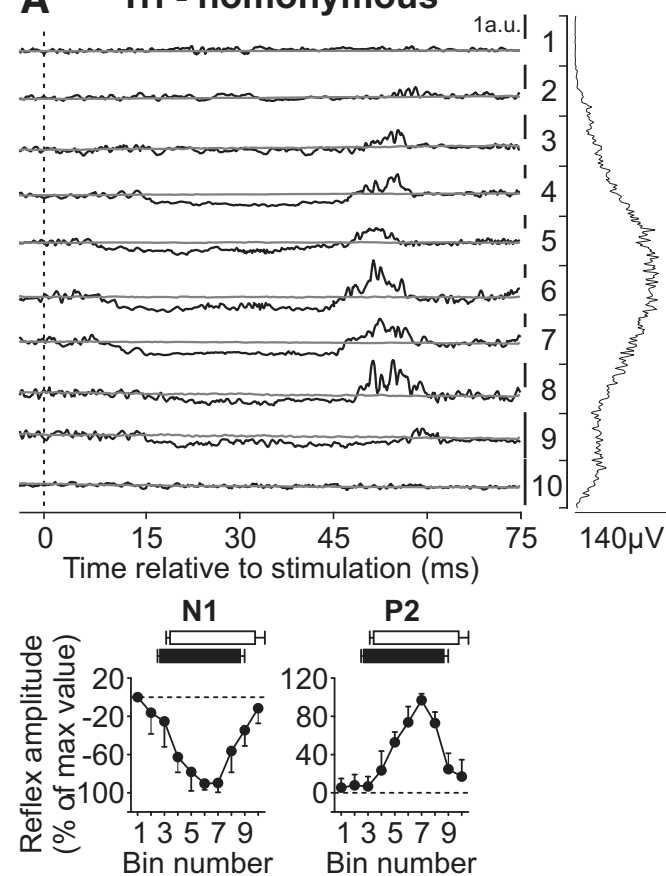

C Tri - homolateral

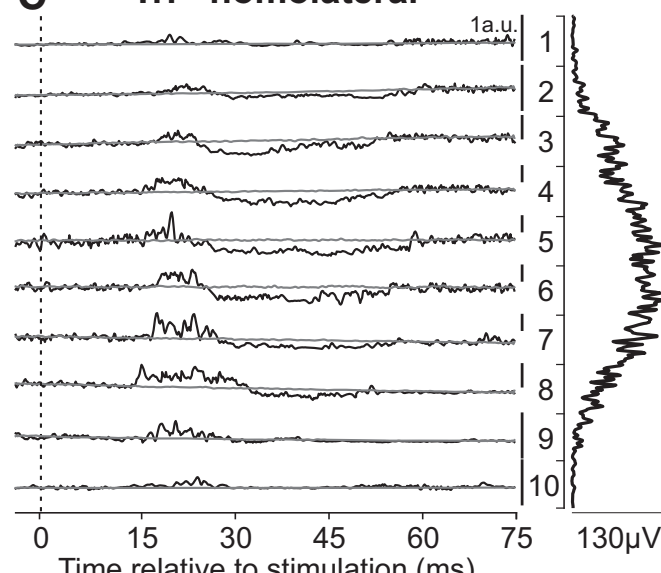

Time relative to stimulation (ms)

P1

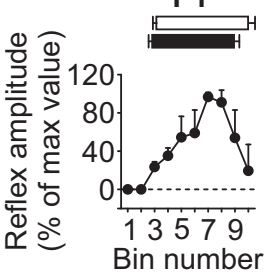

B

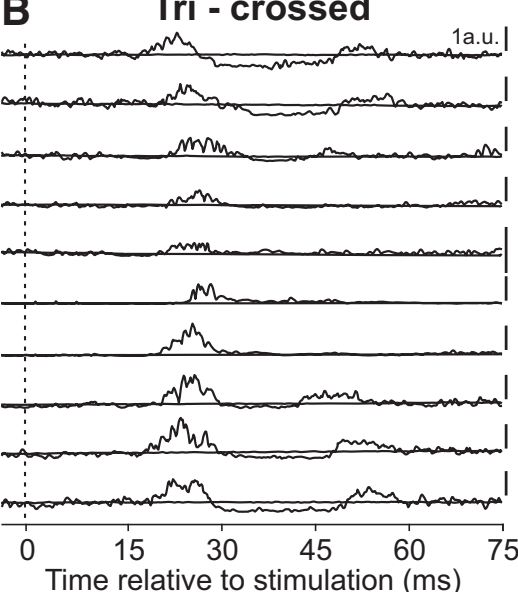

P1
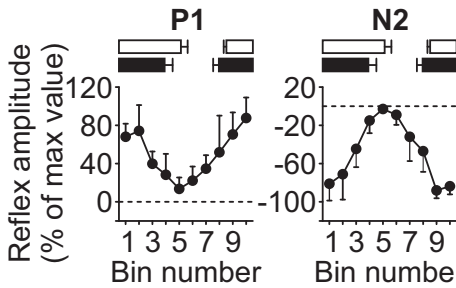

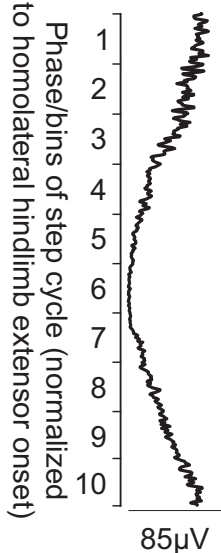

P3

120

\section{$\square$ Stance}

- Muscle activity

\section{Tri - diagonal}
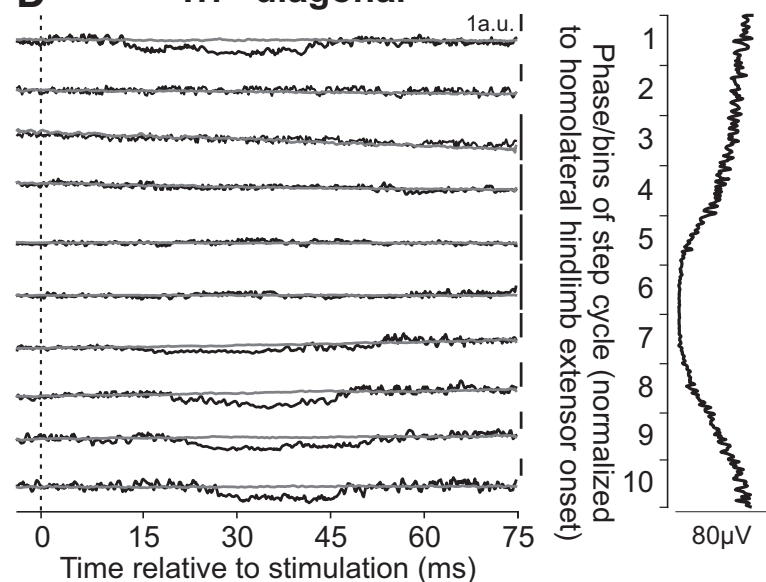

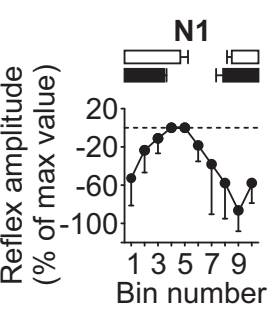

Figure 7. Intralimb and interlimb cutaneous reflexes in Tri. SR nerve stimulations evoked ( $\boldsymbol{A}$ ) homonymous ( 5 trials in 3 cats) and ( $\boldsymbol{B}$ ) crossed responses ( 4 trials in 3 cats), whereas SP nerve stimulations evoked ( $C$ homolateral ( 4 trials in 3 cats) and ( $\boldsymbol{D})$ diagonal ( 5 trials in 3 cats) responses in the Tri. Each panel represents averaged waveforms in the 10 bins of the cycle with a window of 80 ms that starts 5 ms before stimulation in a single cat. Black traces represent averaged cycles that received a stimulation (i.e., reflex responses, $n=6-17$ stimuli per bin). Gray traces represent averaged cycles with no stimuli ( $n=83-146$ cycles). Scale bars are shown in arbitrary units (a.u.) for each bin and can differ to optimize evoked responses. Aligned vertically is the average rectified EMG trace of the Tri muscle over the cycle normalized to a hindlimb extensor on the side of the stimulation. Scatter plots represent reflex amplitudes (mean \pm SD) in each bin of the cycle expressed as a percentage of the maximal response found in one of the bins for pooled data. Short-latency (P1/N1), mid-latency (P2/N2), and longer-latency (P3) responses are shown separately.

muscle was inactive (Fig. $8 B$ ). The crossed P1, N2/P2, and P3 responses in ECU were all significantly modulated by phase for pooled data $(p=0.0002, p=0.045$, and $p=0.014$, respectively, ANOVA).
Responses in ECU evoked by stimulating hindlimb nerves have not been described during cat locomotion. Stimulating the SP nerve evoked similar response patterns in the homolateral (Fig. 8C) and diagonal (Fig. 8D) ECU muscles that consisted of 


\section{A ECU - homonymous}
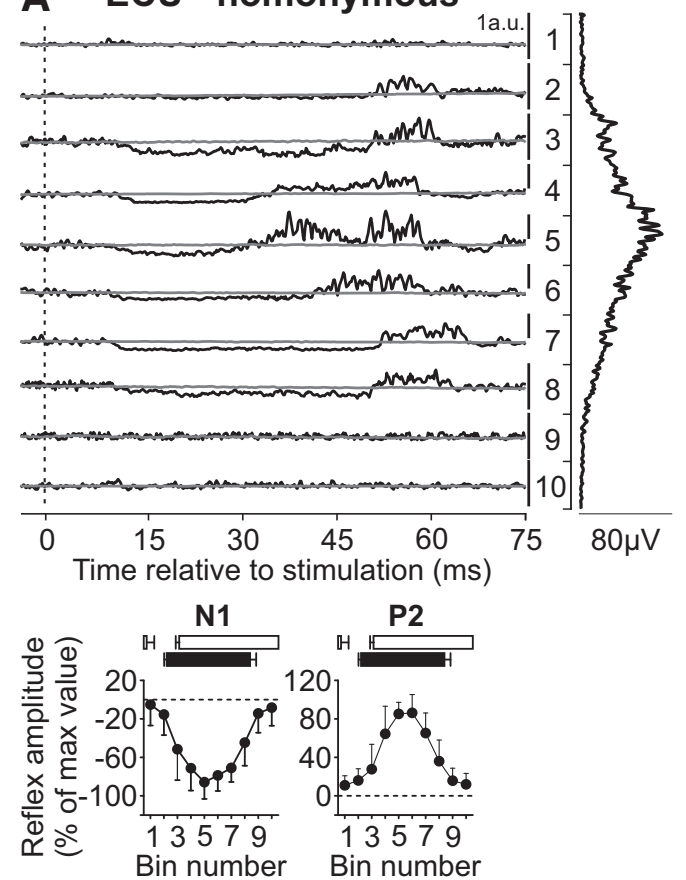

C ECU - homolateral

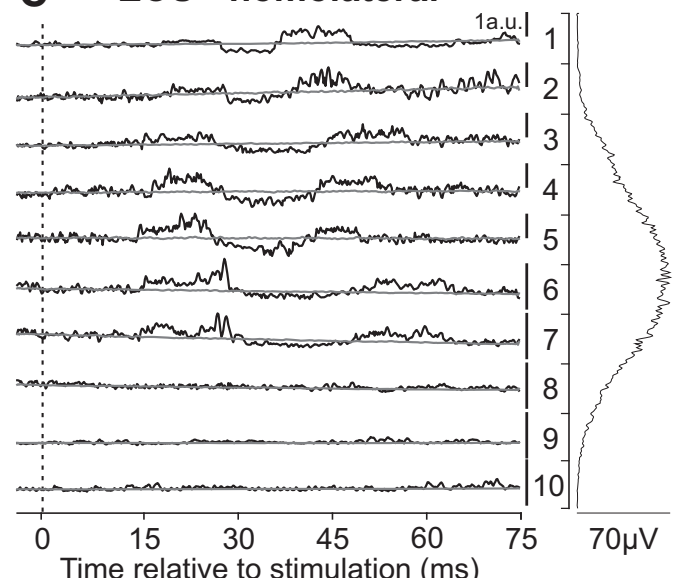

P1

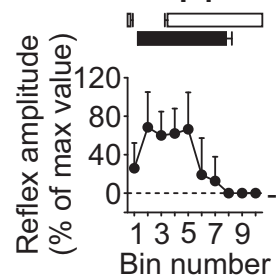

N2

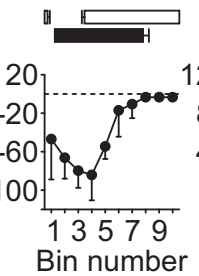

P3

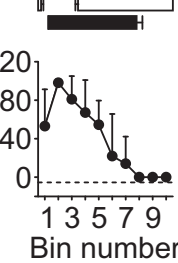

\section{B ECU - crossed}
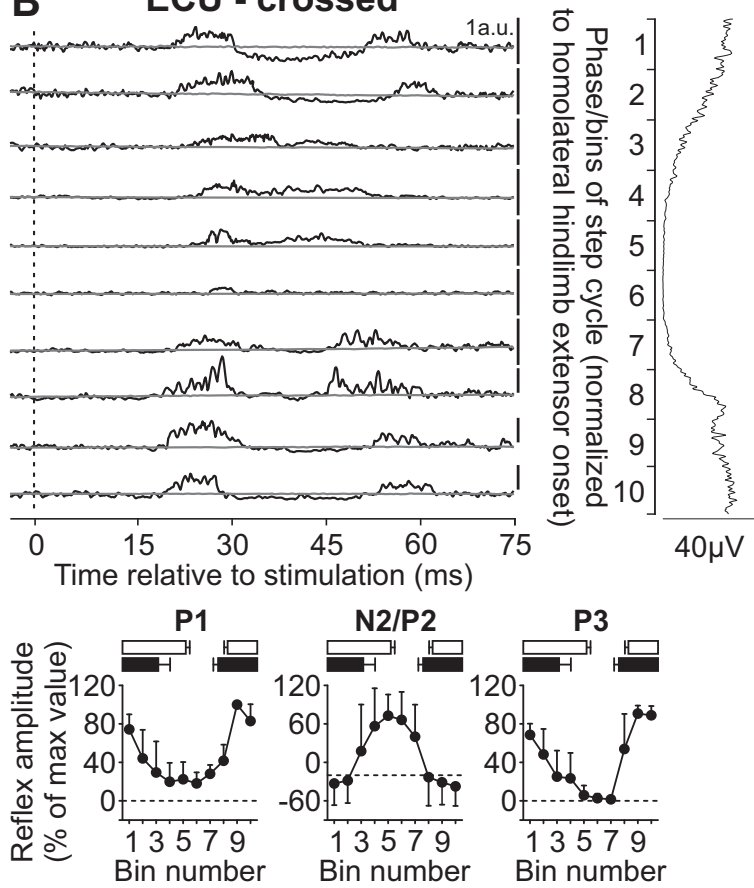

Bin number

$\square$ Stance

- Muscle activity

\section{ECU - diagonal}
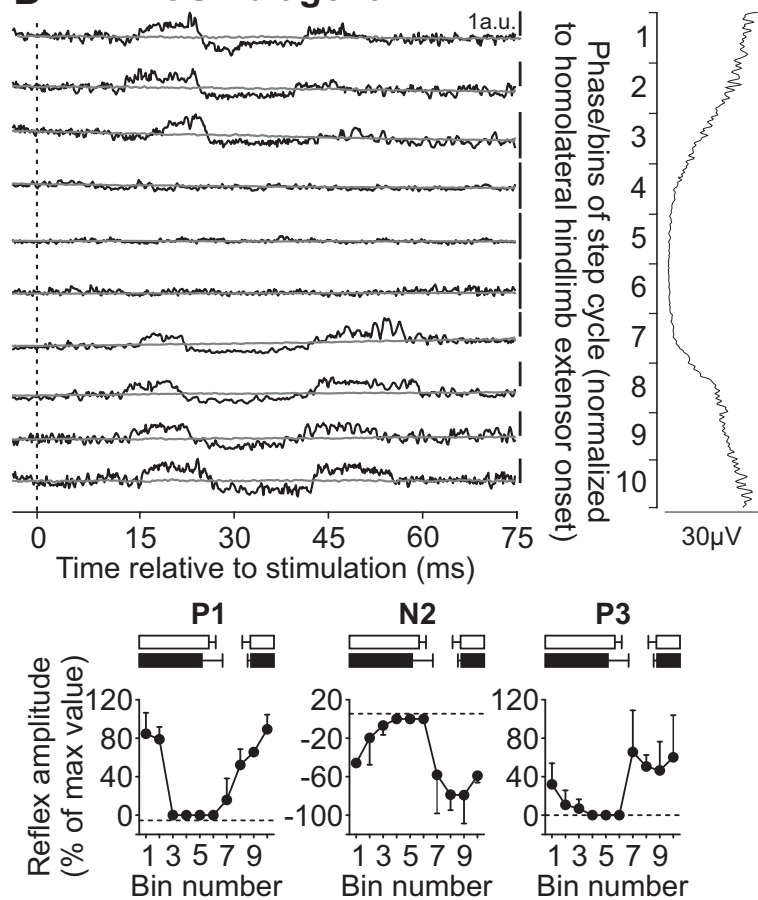

Figure 8. Intralimb and interlimb cutaneous reflexes in ECU. SR nerve stimulations evoked ( $\boldsymbol{A}$ ) homonymous ( 5 trials in 3 cats) and (B) crossed responses ( 5 trials in 3 cats), whereas SP nerve stimulations evoked ( $\boldsymbol{C}$ homolateral ( 4 trials in 3 cats) and $(\boldsymbol{D})$ diagonal ( 5 trials in 3 cats) responses in the ECU. Each panel represents averaged waveforms in the 10 bins of the cycle with a window of 80 ms that starts 5 ms before stimulation in a single cat. Black traces represent averaged cycles that received a stimulation (i.e., reflex responses, $n=5-19$ stimuli per bin). Gray traces represent averaged cycles with no stimuli ( $n=83-146$ cycles). Scale bars are shown in arbitrary units (a.u.) for each bin and can differ to optimize evoked responses. Aligned vertically is the average rectified EMG trace of the ECU muscle over the cycle normalized to a hindlimb extensor on the side of the stimulation. Scatter plots represent reflex amplitudes (mean \pm SD) in each bin of the cycle expressed as a percentage of the maximal response found in one of the bins for pooled data. Short-latency (P1/N1), mid-latency (P2/N2), and longer-latency (P3) responses are shown separately.

$\mathrm{P} 1$ responses followed by $\mathrm{N} 2$ and $\mathrm{P} 3$ responses, primarily when the muscle was active. The homolateral and diagonal P1, N2, and $\mathrm{P} 3$ responses in ECU were significantly modulated by phase for pooled data (homolateral: $p=0.020, p=0.016$, and $p=0.005$, respectively; diagonal: $p=0.002, p=0.047$, and $p=0.024$, respectively, ANOVA).

In summary, inputs from all four limbs evoked responses in a given forelimb muscle, including muscles that flex (BB) or extend 
Table 1. Strength of first excitatory reflex response ${ }^{a}$

\begin{tabular}{cclll}
\hline Muscle & Homonymous (\%) & Crossed (\%) & Homolateral (\%) & Diagonal (\%) \\
\hline Hindlimb & & & & \\
$\quad$ St & $1167 \pm 541$ & $355 \pm 155$ & $300 \pm 111$ & $423 \pm 184$ \\
Srt & $420 \pm 173$ & $183 \pm 25$ & $212 \pm 69$ & $283 \pm 87$ \\
VL & $228 \pm 73$ & $195 \pm 72$ & $235 \pm 19$ & $209 \pm 26$ \\
LG & $388 \pm 149$ & $176 \pm 33$ & $166 \pm 17$ & $206 \pm 43$ \\
Forelimb & & & & \\
BB & $804 \pm 633$ & $339 \pm 158$ & $168 \pm 48$ & $161 \pm 19$ \\
Tri & $243 \pm 64$ & $396 \pm 195$ & $149 \pm 63$ & - \\
ECU & $273 \pm 76$ & $286 \pm 137$ & $140 \pm 15$ & $143 \pm 11$ \\
\hline
\end{tabular}

${ }^{a}$ In each muscle and for each type of response, the amplitude of the first evoked excitatory response (P1 or P2) was expressed as a percentage of the control background level of activity during the muscle's peak period of activity. The number of responses pooled for each muscle/response type is indicated in Table 2 . Each value is the mean \pm SD for pooled data.

(Tri) the elbow and shoulder, and extend (ECU) the wrist. The pattern of evoked responses depended on the muscle and the input limb. The majority of responses evoked in forelimb muscles were modulated with phase.

\section{Variability in reflex responses evoked by inputs from the four limbs}

Based on electrophysiological (Juvin et al., 2012) and anatomical (Brockett et al., 2013) results in rats, it was proposed that the hindlimbs exert a stronger effect on circuits controlling the forelimb than those from the forelimb to the hindlimb. However, in decerebrate cats, Miller et al. (1977) reported that stimulating a forelimb cutaneous nerve generated stronger responses in hindlimb muscles compared with those evoked in forelimb muscles by hindlimb cutaneous nerve stimulation. To estimate the strength of cutaneous reflex pathways, we used two approaches. First, we measured the reflex response as a percentage of the control background level of activity for the first evoked excitatory response ( $\mathrm{P} 1$ or $\mathrm{P} 2)$ in each muscle during the muscle's peak period of activity (Table 1). For instance, homonymous responses in St were $1167 \pm 541 \%$, or over 11 times, greater than the background level of EMG activity during the St muscle's peak period of activity. For pooled data of responses evoked by SP nerve stimulation, homonymous responses were significantly greater than crossed ( $p<0.0001$, ANOVA), homolateral ( $p=$ 0.001 , ANOVA), and diagonal ( $p<0.0001$, ANOVA) responses (Fig. 9A). For SR nerve stimulation, the only significant difference was between homonymous and homolateral responses $(p=$ 0.039 , ANOVA). There were no significant differences between homolateral and diagonal responses evoked by SP and SR nerve stimulation.

Second, we measured the probability that a nerve stimulation elicited homonymous, crossed, homolateral, or diagonal responses for each muscle (Table 2. For each muscle, the number of trials where $\mathrm{P} 1, \mathrm{P} 2, \mathrm{P} 3, \mathrm{~N} 1$, or $\mathrm{N} 2$ responses were observed is represented as a fraction of the total number of trials, with the percentage indicated in parentheses. Table 2 shows that homonymous responses in flexors (St, Srt, BB) had the highest percentages. This is expected as the stimulation threshold was based on responses in these muscles. Some responses were infrequent, such as crossed responses in St $(33 \%)$ and diagonal responses in $\mathrm{BB}$ $(30 \%)$. To determine whether there was a greater percentage of responses evoked by SP or SR nerve stimulations, we pooled the different types of responses evoked by these two nerve stimulations. Overall, there was a significantly greater percentage of responses evoked by SR nerve stimulation for all types of responses (homonymous, $p=0.049$; crossed, $p=0.034$; homolateral, $p=$
0.045; and diagonal, $p=0.048$, ANOVA) (Fig. 9B), consistent with the hypothesis that pathways activated by forelimb cutaneous inputs are stronger or more distributed than those from hindlimb cutaneous inputs.

\section{Latencies of cutaneous reflex responses evoked by inputs from the four limbs}

In studies of intracellular motoneuronal recordings, the latencies of inhibitory or excitatory post-synaptic potentials in relation to cord dorsum potentials can be used to estimate the number of synapses between the primary afferent and the motoneuron (Degtyarenko et al., 1998; Burke et al., 2001; Quevedo et al., 2005b). Although our EMG recordings do not have the same resolution as intracellular recordings, measuring and comparing the latencies of the first observed responses ( $\mathrm{P} 1, \mathrm{P} 2$, or N1) provides a rough estimate of the number of synaptic contacts in the reflex pathways from the four limbs. Table 3 shows the latencies of the first observed homonymous, crossed, homolateral, and diagonal responses for each muscle. As expected, homonymous responses had the shortest latencies. However, for several muscles, homolateral and diagonal responses had shorter latencies than crossed responses, with no apparent differences between homolateral and diagonal responses. Thus, we pooled data for the different types of responses evoked by SP and SR nerve stimulation for statistical analysis (Fig. 9C). Homolateral responses evoked by SP nerve stimulation had significantly shorter latencies than those evoked by SR nerve stimulation $(p<0.0001$, ANOVA). Similar results were found for diagonal responses $(p<$ 0.0001, ANOVA). Homonymous responses evoked by SP nerve stimulation had significantly shorter latencies than homolateral and diagonal responses evoked by the same nerve $(p=0.043$ and 0.049, ANOVA). Homonymous responses evoked by SR nerve stimulation had significantly shorter responses than the other three responses evoked by the same nerve (crossed, $p<0.0001$; homolateral, $p<0.0001$; and diagonal, $p<0.0001$, ANOVA). The latencies of crossed responses evoked by SP nerve stimulation were not significantly different from the other responses due to their high variance. For both nerve stimulations, there was no significant differences in the latencies of crossed, homolateral, and diagonal responses, consistent with a similar number of synaptic relays in their pathways.

\section{Effect of stimulating cutaneous nerves on extensor burst durations and onsets}

Stimulating cutaneous nerves during real or fictive locomotion can modify the timing and duration of specific bursts to maintain interlimb coordination (Duysens and Stein, 1978; Guertin et al., 1995; Frigon and Rossignol, 2008b). To determine whether stimulating the SP and SR nerves altered the locomotor pattern, we measured extensor burst durations in all four limbs (Tri and VL for the forelimbs and hindlimbs, respectively) when stimulation was applied during mid-stance or mid-swing of the input leg (homonymous limb). With stimulation during mid-swing, we measured the duration of the following homonymous extensor burst and its onset.

When the SP nerve was stimulated during mid-stance of the homonymous hindlimb, the crossed hindlimb was in mid-swing while the homolateral and diagonal forelimbs were in early and late stance, respectively (Fig. 10A, bottom). Burst durations were significantly prolonged in the homonymous VL $(p=0.000003$, ANOVA) and homolateral Tri ( $p=0.0005$, ANOVA) (Fig. $10 A$, top). When the SP nerve was stimulated during mid-swing of the homonymous hindlimb, the crossed hindlimb was in mid-stance 

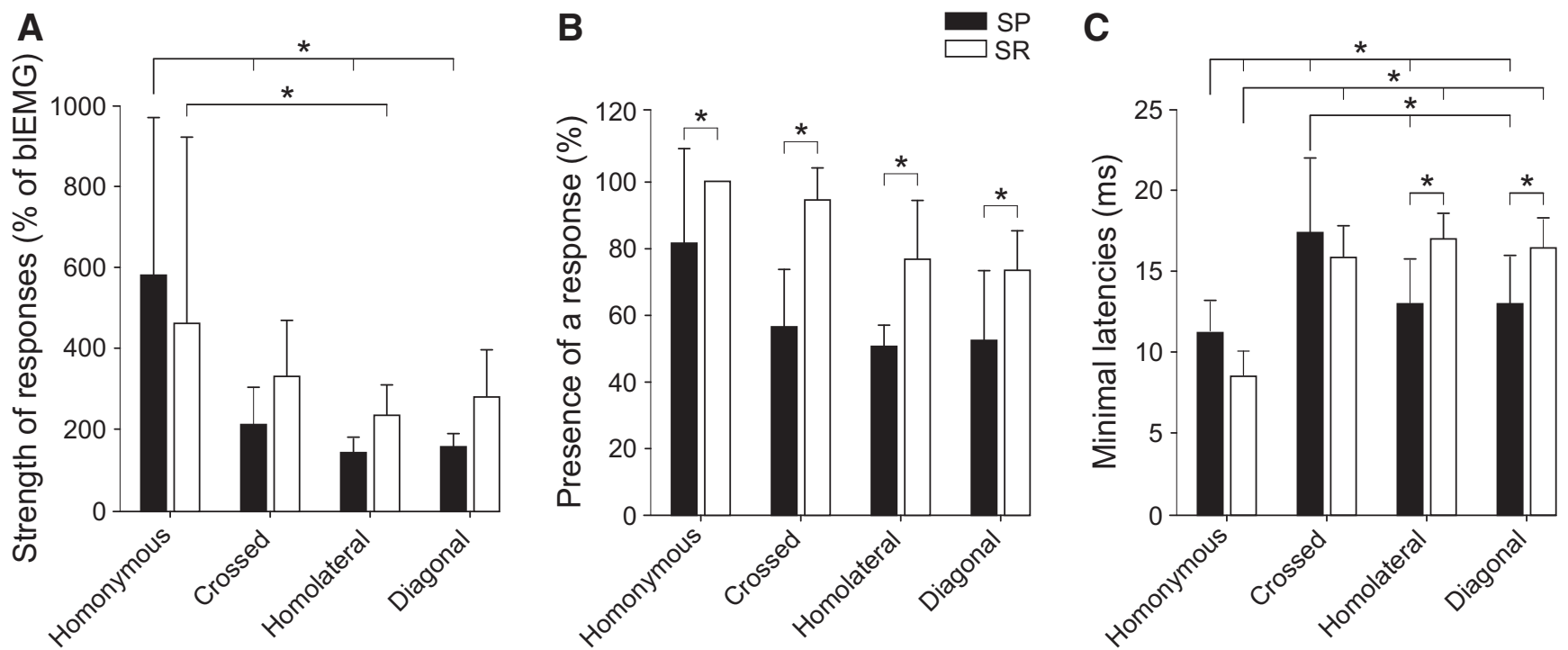

Figure 9. Relative strengths, presence, and latencies of reflex responses. $A$, For each type of response evoked by SP or SR nerve stimulation, the amplitude of the first evoked excitatory response ( $\mathrm{P} 1$ or P2) was expressed as a percentage of the control background level of activity during the muscle's peak period of activity. These data were pooled for each type of response evoked by SP and SR nerve stimulation. $\boldsymbol{B}$, The percentage of trials where any type of response was observed in a given muscle was measured. Data were pooled for each type of response evoked by SP and SR nerve stimulation. C, The latencies of the first observed homonymous, crossed, homolateral, and diagonal responses were measured for each muscle. Data were pooled for each type of response evoked by SP and SR nerve stimulation. $\boldsymbol{A}-\boldsymbol{C}$, Error bar indicates mean \pm SD of pooled data. ${ }^{*} p<0.05$, significant differences between responses evoked by SP and SR nerve stimulation.

Table 2. Presence of responses evoked by cutaneous inputs from the four limbs

\begin{tabular}{|c|c|c|c|c|c|}
\hline \multirow[b]{2}{*}{ Muscle } & \multicolumn{5}{|c|}{ Reflex responses } \\
\hline & P1 & P2 & P3 & N1 & N2 \\
\hline Semitendinosus-Homonymous & $10 / 10(100 \%)$ & $10 / 10(100 \%)$ & - & - & - \\
\hline Semitendinosus-Crossed & - & $3 / 9(33 \%)$ & - & - & - \\
\hline Semitendinosus-Homolateral & $4 / 5(80 \%)$ & $2 / 5(40 \%)$ & - & - & $2 / 5(40 \%)$ \\
\hline Semitendinosus-Diagonal & $3 / 5(60 \%)$ & & - & - & - \\
\hline Sartorius-Homonymous & - & 10/10 (100\%) & - & 10/10 (100\%) & - \\
\hline Sartorius-Crossed & - & $6 / 10(60 \%)$ & - & $6 / 10(60 \%)$ & - \\
\hline Sartorius-Homolateral & $5 / 5(100 \%)$ & - & $4 / 5(80 \%)$ & - & $5 / 5(100 \%)$ \\
\hline Sartorius-Diagonal & $5 / 6(83 \%)$ & - & $3 / 6(50 \%)$ & - & $5 / 6(83 \%)$ \\
\hline Vastuslateralis-Homonymous & $6 / 7(86 \%)$ & $6 / 7(86 \%)$ & - & $6 / 7(86 \%)$ & - \\
\hline Vastuslateralis-Crossed & - & $6 / 8(75 \%)$ & - & - & - \\
\hline Vastus lateralis-Homolateral & $3 / 5(60 \%)$ & - & $2 / 5(40 \%)$ & - & $2 / 5(40 \%)$ \\
\hline Vastus lateralis-Diagonal & $5 / 6(83 \%)$ & - & $4 / 6(67 \%)$ & - & $5 / 6(83 \%)$ \\
\hline Lateral gastrocnemius-Homonymous & $4 / 10(40 \%)$ & $4 / 10(40 \%)$ & - & 4/10 (40\%) & - \\
\hline Lateral gastrocnemius-Crossed & - & 4/7 (57\%) & - & 3/7 (43\%) & - \\
\hline Lateral gastrocnemius-Homolateral & $2 / 3(67 \%)$ & - & $2 / 3(67 \%)$ & - & $2 / 3(67 \%)$ \\
\hline Lateral gastrocnemius-Diagonal & $2 / 3(67 \%)$ & - & $2 / 3(67 \%)$ & - & $2 / 3(67 \%)$ \\
\hline Biceps brachii-Homonymous & $5 / 6(83 \%)$ & $6 / 6(100 \%)$ & - & - & - \\
\hline Biceps brachii-Crossed & $5 / 6(83 \%)$ & - & - & - & - \\
\hline Biceps brachii-Homolateral & - & $5 / 10(50 \%)$ & - & $5 / 10(50 \%)$ & - \\
\hline Biceps brachii-Diagonal & $3 / 10(30 \%)$ & - & - & - & $3 / 10(30 \%)$ \\
\hline Triceps brachii-Homonymous & - & $5 / 5(100 \%)$ & - & $5 / 5(100 \%)$ & - \\
\hline Triceps brachii-Crossed & $3 / 4(75 \%)$ & - & $3 / 4(75 \%)$ & - & 4/4 (100\%) \\
\hline Triceps brachii-Homolateral & $2 / 7(29 \%)$ & - & - & - & 4/7 (57\%) \\
\hline Triceps brachii-Diagonal & - & - & - & $5 / 7(71 \%)$ & - \\
\hline Extensor carpi ulnaris-Homonymous & - & $5 / 5(100 \%)$ & - & $5 / 5(100 \%)$ & - \\
\hline Extensor carpi ulnaris-Crossed & $5 / 5(100 \%)$ & $5 / 5(100 \%)$ & $3 / 5(60 \%)$ & - & $4 / 5(80 \%)$ \\
\hline Extensor carpi ulnaris-Homolateral & $4 / 9(44 \%)$ & - & $4 / 9(44 \%)$ & - & $4 / 9(44 \%)$ \\
\hline Extensor carpi ulnaris-Diagonal & $5 / 9(56 \%)$ & - & $5 / 9(56 \%)$ & - & $5 / 9(56 \%)$ \\
\hline
\end{tabular}


Table 3. Latencies of first observed responses evoked by cutaneous inputs from the four limbs ${ }^{a}$

\begin{tabular}{lcccr}
\hline & \multicolumn{4}{c}{ Latencies of first observed responses (ms) } \\
\cline { 2 - 5 } Muscle & Homonymous & \multicolumn{1}{l}{ Crossed } & Homolateral & \multicolumn{1}{c}{ Diagonal } \\
\hline St & $9.8 \pm 1.8$ & $24.3 \pm 1.2$ & $17.5 \pm 2.1$ & $17.5 \pm 2.1$ \\
Srt & $11.9 \pm 2.0$ & $13.7 \pm 1.0$ & $17.5 \pm 1.7$ & $17.5 \pm 1.7$ \\
VL & $12.2 \pm 1.3$ & $20.0 \pm 3.7$ & $15.3 \pm 0.6$ & $15.7 \pm 0.6$ \\
LG & $11.2 \pm 2.1$ & $14.5 \pm 0.6$ & $16.5 \pm 0.7$ & $16 \pm 1.4$ \\
BB & $7.8 \pm 1.1$ & $14.8 \pm 1.1$ & $14 \pm 3.6$ & $14 \pm 2.6$ \\
Tri & $8.6 \pm 1.5$ & $16.3 \pm 1.5$ & $13.5 \pm 3.9$ & $14 \pm 4.2$ \\
ECU & $9.2 \pm 1.9$ & $16.6 \pm 2.7$ & $11.5 \pm 0.7$ & $12 \pm 1.4$ \\
\hline
\end{tabular}

${ }^{a}$ The latencies of the first observed homonymous, crossed, homolateral, and diagonal responses were measured for each muscle.

while the homolateral and diagonal forelimbs were in late and early stance, respectively (Fig. 10A, bottom). The VL burst of the homonymous hindlimb was significantly delayed by $7.50 \pm$ $2.17 \%$ ( $p=0.00006$, ANOVA, data not shown). Only the VL burst of the crossed hindlimb was significantly prolonged ( $p=$ 0.0003, ANOVA) (Fig. 10A, top).

When the SR nerve was stimulated during mid-stance of the homonymous forelimb, the crossed forelimb was in mid-swing while the homolateral and diagonal hindlimbs were in late and early stance, respectively (Fig. 10B, bottom). Burst durations were significantly prolonged in the homonymous Tri $(p=$ 0.0003 , ANOVA $)$ as well as in the homolateral $(p=0.0003$, ANOVA) and diagonal ( $p=0.001$ ) VL (Fig. 10B, top). When the SR nerve was stimulated during mid-swing of the homonymous forelimb, the crossed forelimb was in mid-stance while the homolateral and diagonal hindlimbs were in early and late stance, respectively (Fig. 10B, bottom). The Tri burst of the homonymous forelimb was significantly delayed by $8.44 \pm 0.33 \%(p=$ 0.00000002 , ANOVA, data not shown). The crossed Tri ( $p=$ 0.00007 , ANOVA), homolateral VL ( $p=0.00002$, ANOVA), and diagonal VL $(p=0.00005$, ANOVA) were significantly prolonged (Fig. 10B, top). These results indicate that hindlimb and forelimb cutaneous inputs modify the duration and/or timing of extensor muscles in all four limbs.

\section{Discussion}

Sherrington (1906) proposed that reflex pathways interconnected different levels of the nervous system. Here, we characterized cutaneous reflexes in all four limbs during quadrupedal locomotion, showing that, when cutaneous inputs from paw dorsa enter the spinal cord, they affect circuits controlling all four limbs. These reflex responses included short- (P1/N1), mid- (P2/ $\mathrm{N} 2$ ), and longer-latency (P3) excitatory or inhibitory responses that were phase-modulated. We discuss the role of these responses in the control of locomotion and potential neural mechanisms/pathways involved.

\section{Functional considerations}

The SP and SR nerves supply the paw dorsa, and their afferents are normally activated when contacting an obstacle (Bernard et al., 2007). As in Figure 10, we discuss the potential function of these responses with stimuli applied during mid-swing and midstance of the homonymous hindlimb and forelimb, with SP and SR nerve stimulation, respectively. Stimulating the SP nerve during hindlimb mid-swing elicited responses consistent with lifting the homonymous hindlimb away (P1 responses in St, LG; both knee flexors) and over the simulated contact while coactivating crossed hindlimb flexors and extensors (in mid-stance), possibly to increase limb stiffness for optimal support. Homonymous VL burst onset was delayed, whereas crossed VL burst duration was prolonged (Fig. 10A). The N1/P2 responses in the homolateral elbow flexor (BB) and N1 responses in the diagonal elbow extensor (Tri) are consistent with a brief arrest of the forelimb pattern. Homolateral and diagonal forelimb Tri burst durations were not affected (Fig. 10A). When the SP nerve was stimulated during homonymous hindlimb mid-stance, there was coactivation of hip flexors (Srt) and extensors (St) and short-latency inhibition followed by excitation in knee (VL) and ankle (LG) extensors of the homonymous hindlimb. The homonymous VL burst was also prolonged (Fig. 10A). Responses in the crossed hindlimb (in mid-swing) and diagonal forelimb (in late stance) were weak. In extensors (Tri, ECU) of the homolateral forelimb (in early stance), P1/N2 responses were evoked, consistent with reinforcing weight support and delaying push-off. In agreement, homolateral Tri burst duration was prolonged (Fig. 10A).

Responses evoked by SR nerve stimulation appear to play similar functional roles. Stimulating the SR nerve during homonymous forelimb mid-swing evoked $\mathrm{P} 1 / \mathrm{P} 2$ responses in the homonymous elbow flexor $\mathrm{BB}$ and crossed $\mathrm{P} 1$ responses in $\mathrm{BB}$, Tri, and ECU. The crossed Tri burst was also prolonged, delaying push-off (Fig. 10B). In the homolateral (in early stance) and diagonal (in late stance) hindlimbs, $\mathrm{P} 1 / \mathrm{N} 2 / \mathrm{P} 3$ responses were evoked, consistent with reinforcing weight support, delaying and facilitating push-off. In agreement, homolateral and diagonal VL burst durations were prolonged (Fig. 10B). Stimulating the SR nerve during homonymous forelimb mid-stance evoked N1/P2 responses in Tri and ECU. In the crossed forelimb (in midswing), weak coactivation of BB, Tri, and ECU was observed, without affecting crossed Tri burst duration (Fig. 10B). In the homolateral (in late stance) and diagonal (early stance) hindlimbs, P1 responses were found in Srt, St, VL, and LG that were followed by $\mathrm{N} 2 / \mathrm{P} 3$ responses in VL and LG, consistent with stabilizing the hindlimbs, delaying and facilitating push-off. In agreement, the homolateral and diagonal VL burst durations were prolonged (Fig. 10B).

To summarize, cutaneous reflexes during mid-swing adjust limb trajectory, mainly by flexing the homonymous limb, and by modifying the support phases of the other three limbs, either by coactivating flexors and extensors and/or by delaying and then facilitating push-off. Cutaneous reflexes during mid-stance stabilize the homonymous limb by prolonging its extension phase and by modifying the support phases of the homolateral and diagonal limbs, either by coactivating flexors and extensors, and/or by delaying and then facilitating push-off. We propose that responses in homolateral and diagonal limbs are a global attempt to initially stabilize the limbs, by coactivating flexors and extensors, followed by briefly arresting ongoing extensor activity before producing a meaningful late response.

\section{Neural pathways and mechanisms involved in reflex responses}

The present study showed excitatory and inhibitory responses in all four limbs with SP or SR nerve stimulation, consistent with activation of both excitatory and inhibitory spinal circuits at cervical and lumbar levels bilaterally. At a stimulation intensity of 1.2 times the motor threshold, responses were most likely mediated by large diameter $\mathrm{A} \beta$ afferents (Drew and Rossignol, 1987; Pratt et al., 1991; LaBella et al., 1992; Quevedo et al., 2005a,b).

The P1 responses latencies in the four limbs are consistent with trisynaptic pathways and are illustrated for left SP nerve stimulation in Figure 11A. In the homonymous forelimbs and 

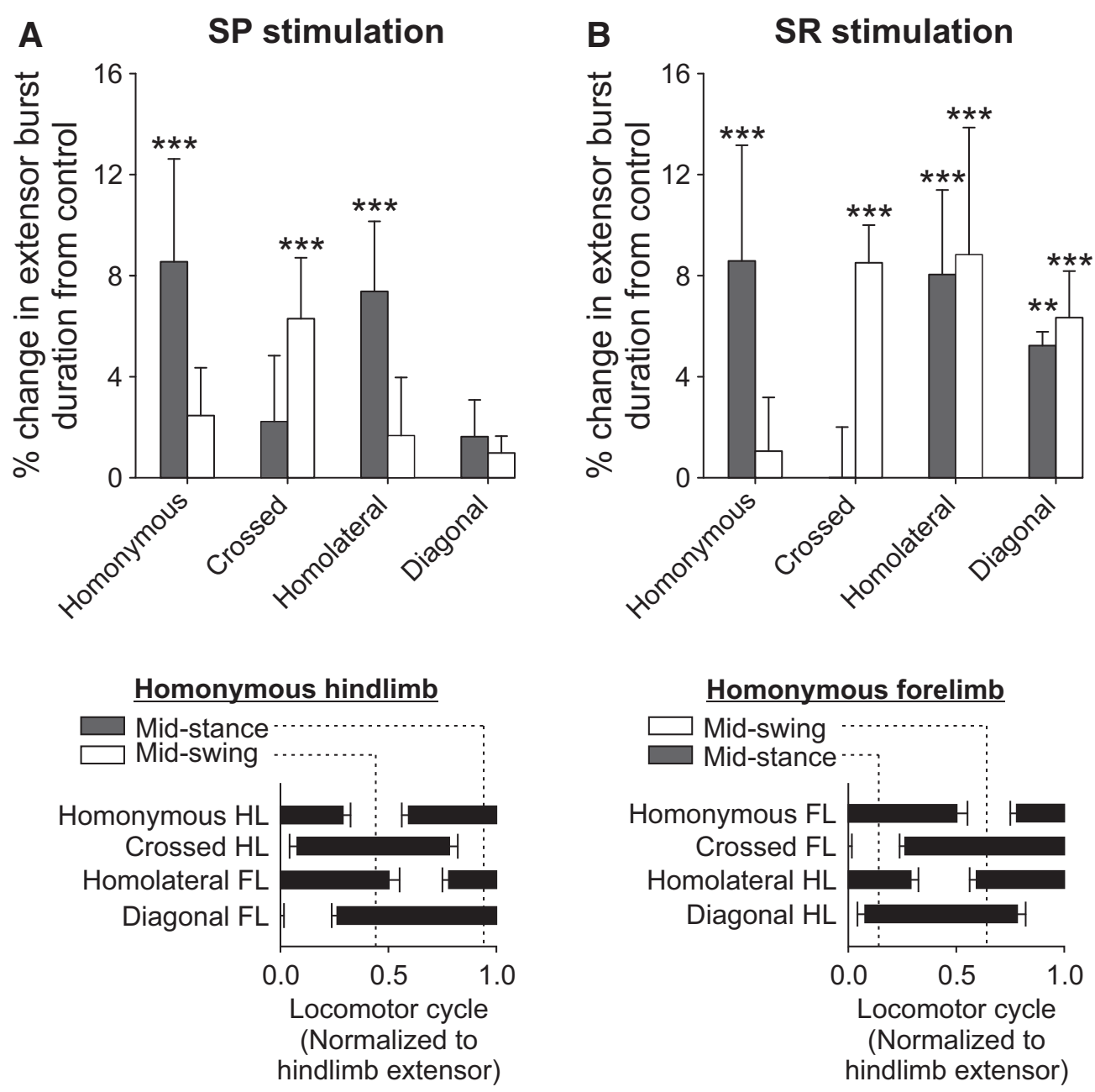

Figure 10. Effect of nerve stimulation on extensor burst durations. Top, The effect of $(\boldsymbol{A})$ SP and $(\boldsymbol{B})$ SR nerve stimulations on extensor burst durations during mid-stance and mid-swing of the input limb (homonymous limb). With stimulation during mid-swing, we measured the duration of the following homonymous extensor burst and its onset. We measured Tri ( $n=3-6$ ) and VL ( $n=$ 4 or 5 ) burst durations for the forelimbs and hindlimbs, respectively. Burst durations within a stimulated cycle were expressed as a percentage change from control bursts. Error bars indicate mean \pm SD. Significant differences from control (one-factor ANOVA): ${ }^{* *} p<0.01 ;{ }^{* * *} p<0.001$. Bottom, The mid-stance and mid-swing periods of the homonymous $(\boldsymbol{A})$ hindlimb and $(\boldsymbol{B})$ forelimb in relation to the other limbs. Data are taken from Figure $1 B$.

hindlimbs, we observed P1 responses at $\sim 8-10 \mathrm{~ms}$ (Table 3 ). During fictive locomotion in curarized decerebrate cats, shortlatency homonymous responses in forelimb and hindlimb motoneurons with SR and SP nerve stimulation, respectively, were consistent with trisynaptic pathways (Hishinuma and Yamaguchi, 1989; Seki and Yamaguchi, 1997; Quevedo et al., 2005b). We observed crossed $\mathrm{N} 1$ hindlimb responses at latencies $<18 \mathrm{~ms}$, the minimal latency for spino-bulbo-spinal reflexes from the hindlimbs in the cat (Shimamura and Livingston, 1963), suggesting that crossed inhibition does not require a supraspinal contribution. Crossed hindlimb responses from cutaneous afferents are minimally trisynaptic and are thought to be mediated by spinal commissural interneurons located in laminae VII and VII, as these afferents terminate in the ipsilateral dorsal horn without direct contralateral projections (Edgley and Wallace, 1989; Edgley and Aggelopoulos, 2006).

In homolateral and diagonal limbs, the first responses evoked by SP or SR nerve stimulation had latencies as short as $\sim 12 \mathrm{~ms}$ and were all $<18 \mathrm{~ms}$ (Table 3 ). These were likely mediated by disynaptic or trisynaptic spinal pathways, as their latencies were not significantly different from the shortest crossed responses, despite greater distance between the cervical and contralateral lumbar side, suggesting fast-conducting pathways (Fig. 11A). Our minimal latencies of $\sim 12 \mathrm{~ms}$ are shorter than those reported by Miller et al. (1977) at $\sim 18 \mathrm{~ms}$, which can be explained by differences in preparation (intact vs decerebrate) and recorded muscles. Using selective spinal lesions, Miller et al. (1977) showed that homolateral responses were mediated by pathways ascending or descending in the ventrolateral spinal cord, where long propriospinal neurons are located (for review, see Flynn et al., 2011; Frigon, 2017). Stimulating forelimb cutaneous afferents, SR nerve included, at rest and during pharmacologically evoked fictive locomotion, evoked homolateral and diagonal responses that were disynaptic or trisynaptic, with a mix of excitatory and inhibitory post-synaptic potentials in decerebrate cats spinal-transected at upper cervical levels (Schomburg et al., 1977, 1978, 1986).

The P2, N2, and P3 responses in the four limbs most likely involve supraspinal contributions. Homonymous $\mathrm{P} 2$ responses are generally reduced or absent following incomplete or complete spinal lesions in cats (Fuwa et al., 1991; Frigon and Rossignol, 2008a; Frigon et al., 2009; Hurteau et al., 2017), as are mid- or longer-latency homolateral responses in high spinal cats (Miller et al., 1977). The bulbospinal reticular formation contains cells that project to the forelimbs and hindlimbs (Shimamura and 
A

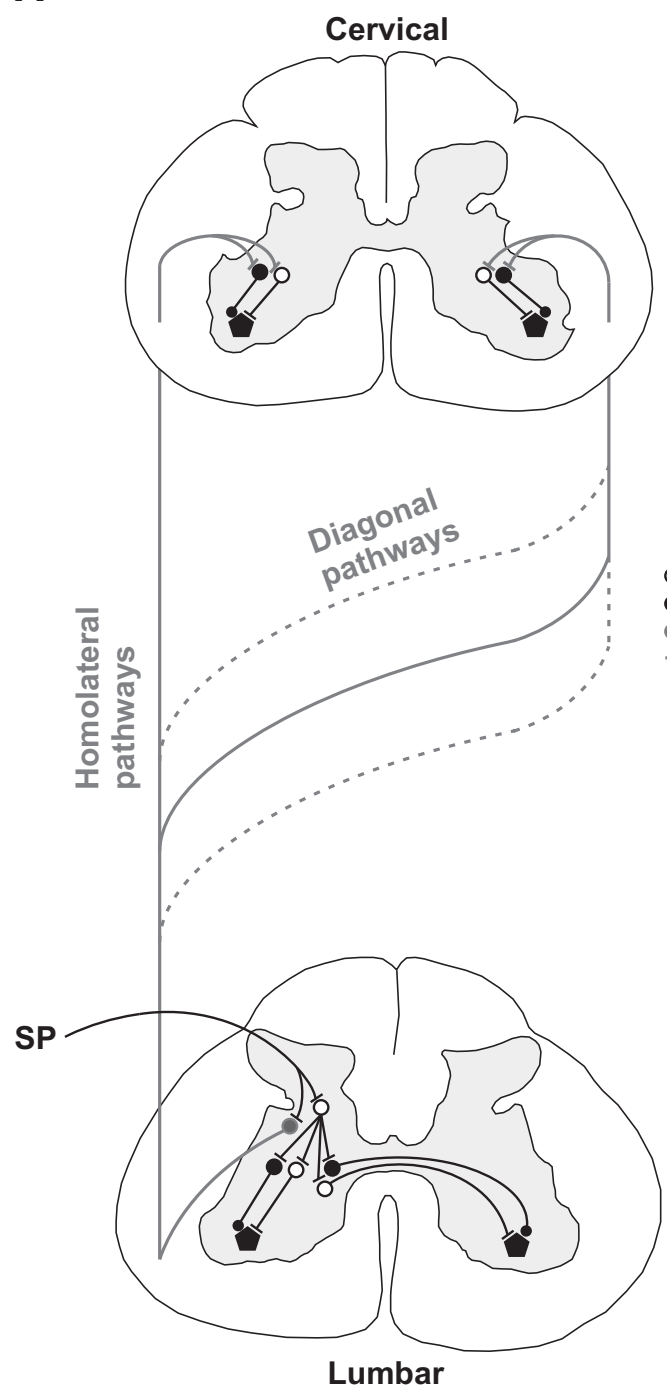

B

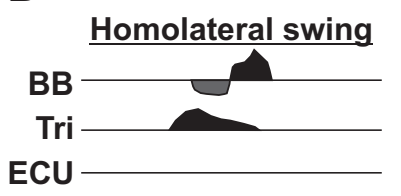

Homolateral stance

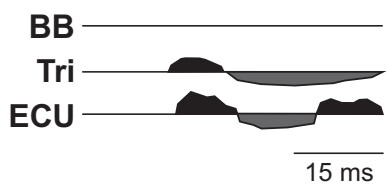

O-1 Excitatory

$\rightarrow$ Inhibitory

- Long propriospinal

- Collateral

Motoneuron

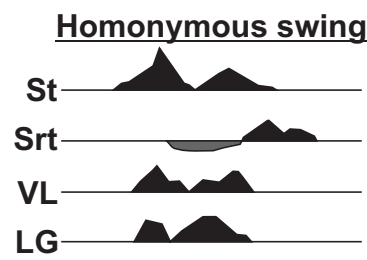

Homonymous stance

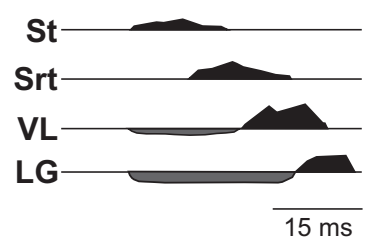

\section{Diagonal swing}

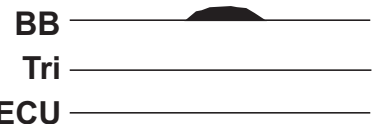

Diagonal stance

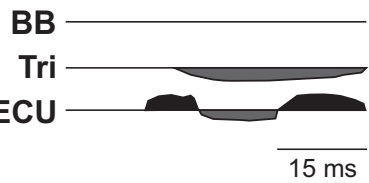

Crossed swing
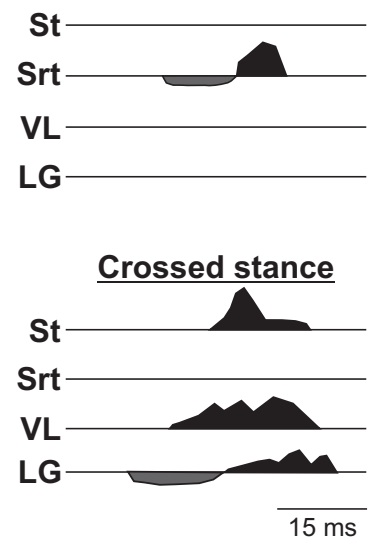

Figure 11. Summary diagram of reflex pathways and main responses during stance and swing evoked by SP nerve stimulation. $\boldsymbol{A}$, Upon entering the spinal cord, primary afferents from the SP nerve contact interneurons that project within the hemisegment (homonymous responses), commissural interneurons that project contralaterally (crossed responses), and long ascending propriospinal neurons with their main axonal projections terminating ipsilaterally (homolateral responses) or on the other side (diagonal responses). Diagonal pathways can cross at various segments along the length of the spinal cord and include collaterals from homolateral pathways that also project contralaterally (Frigon, 2017). B, Each panel represents the main pattern of forelimb and hindlimb responses evoked with SP nerve stimulation when the different limbs are in mid-swing or mid-stance. Responses shaded in black represent excitatory responses. Responses shaded in black represent inhibitory responses. Responses are aligned to the start of the stimulation.

Akert, 1965; Shimamura et al., 1982; Drew and Rossignol, 1984), making it an excellent candidate for interlimb coordination and responses observed in the present study. Cells in the bulbospinal reticular formation are known to contain cells that halt locomotion (Bouvier et al., 2015; Juvin et al., 2016), part of the startle circuit described in animals and humans (Brown et al., 1991; Roseberry and Kreitzer, 2017). Startle responses can be induced by unexpected perturbations (Hof and Duysens, 2018) or loud noises (Nieuwenhuijzen et al., 2000) and are thought to permit an evaluation of external conditions before continuing (Eilam, 2005). It is possible that cells mediating the P2/N2 responses are part of the startle circuit, helping to briefly freeze the pattern to evaluate external conditions.

\section{Strength of ascending and descending reflex pathways}

Descending homolateral and diagonal pathways from the SR nerve had a higher probability of evoking responses or affecting extensor burst durations compared with ascending pathways from the SP nerve (Tables 1, 2; Figs. 9, 10). These results are consistent with those of Miller et al. (1977) of stronger descending pathways from forelimb afferents to hindlimb motor pools, contrasting with stronger ascending influences proposed in rats (Juvin et al., 2012; Thibaudier and Hurteau, 2012; Brockett et al., 2013). As stated, inputs from the SR and SP nerve help to rapidly respond to perturbations. Loss of forelimb support could lead to more serious injuries compared with loss of hindlimb support, such as injury to the head and thoracic organs. Thus, stronger descending pathways from forelimb afferents could reflect a greater protective function.

In conclusion, we showed that cutaneous inputs from the forelimbs and hindlimbs activate motor pools controlling all four limbs during quadrupedal locomotion via fast-conducting spinal pathways, consistent with an important role of these pathways in interlimb coordination. Several questions however remain unan- 
swered. For instance, how are interlimb pathways activated by other inputs during quadrupedal locomotion, such as those normally activated during unobstructed locomotion? How are interlimb reflexes reorganized following spinal cord injury or other lesion types? What is the relative contribution of supraspinal structures in modulating interlimb reflexes and coordination before and after injury? We are currently investigating some of these questions using split-belt locomotion, spinal lesions, and computational modeling to integrate experimental results into a framework for understanding the control of interlimb coordination.

\section{References}

Abraham LD, Marks WB, Loeb GE (1985) The distal hindlimb musculature of the cat. cutaneous reflexes during locomotion. Exp Brain Res 58:594603. CrossRef Medline

Bernard G, Bouyer L, Provencher J, Rossignol S (2007) Study of cutaneous reflex compensation during locomotion after nerve section in the cat. J Neurophysiol 97:4173-4185. CrossRef Medline

Bouvier J, Caggiano V, Leiras R, Caldeira V, Bellardita C, Balueva K, Fuchs A, Kiehn O (2015) Descending command neurons in the brainstem that halt locomotion. Cell 163:1191-1203. CrossRef Medline

Bretzner F, Drew T (2005) Motor cortical modulation of cutaneous reflex responses in the hindlimb of the intact cat. J Neurophysiol 94:673-687. CrossRef Medline

Brockett EG, Seenan PG, Bannatyne BA, Maxwell DJ (2013) Ascending and descending propriospinal pathways between lumbar and cervical segments in the rat: evidence for a substantial ascending excitatory pathway. Neuroscience 240:83-97. CrossRef Medline

Brown P, Rothwell JC, Thompson PD, Britton TC, Day BL, Marsden CD (1991) New observations on the normal auditory startle reflex in man. Brain 114:1891-1902. CrossRef Medline

Buford JA, Smith JL (1993) Adaptive control for backward quadrupedal walking: III. Stumbling corrective reactions and cutaneous reflex sensitivity. J Neurophysiol 70:1102-1114. CrossRef Medline

Burke RE, Degtyarenko AM, Simon ES (2001) Patterns of locomotor drive to motoneurons and last-order interneurons: clues to the structure of the CPG. J Neurophysiol 86:447-462. CrossRef Medline

D’Angelo G, Thibaudier Y, Telonio A, Hurteau MF, Kuczynski V, Dambreville C, Frigon A (2014) Modulation of phase durations, phase variations and temporal coordination of the four limbs during quadrupedal split-belt locomotion in intact adult cats. J Neurophysiol 112:1825-1837. CrossRef Medline

Degtyarenko AM, Simon ES, Norden-Krichmar T, Burke RE (1998) Modulation of oligosynaptic cutaneous and muscle afferent reflex pathways during fictive locomotion and scratching in the cat. J Neurophysiol 79: 447-463. CrossRef Medline

Dietz V, Fouad K, Bastiaanse CM (2001) Neuronal coordination of arm and leg movements during human locomotion. Eur J Neurosci 14:1906-1914. CrossRef Medline

Drew T, Rossignol S (1984) Phase-dependent responses evoked in limb muscles by stimulation of medullary reticular formation during locomotion in thalamic cats. J Neurophysiol 52:653-675. CrossRef Medline

Drew T, Rossignol S (1985) Forelimb responses to cutaneous nerve stimulation during locomotion in intact cats. Brain Res 329:323-328. CrossRef Medline

Drew T, Rossignol S (1987) A kinematic and electromyographic study of cutaneous reflexes evoked from the forelimb of unrestrained walking cats. J Neurophysiol 57:1160-1184. CrossRef Medline

Duysens J, Loeb GE (1980) Modulation of ipsi- and contralateral reflex responses in unrestrained walking cats. J Neurophysiol 44:1024-1037. CrossRef Medline

Duysens J, Stein RB (1978) Reflexes induced by nerve stimulation in walking cats with implanted cuff electrodes. Exp Brain Res 32:213-224. Medline

Duysens J, Tax AA, Trippel M, Dietz V (1993) Increased amplitude of cutaneous reflexes during human running as compared to standing. Brain Res 613:230-238. CrossRef Medline

Edgley SA, Aggelopoulos NC (2006) Short latency crossed inhibitory reflex actions evoked from cutaneous afferents. Exp Brain Res 171:541-550. CrossRef Medline
Edgley SA, Wallace NA (1989) A short-latency crossed pathway from cutaneous afferents to rat hindlimb motoneurones. J Physiol 411:469-480. CrossRef Medline

Eilam D (2005) Die hard: a blend of freezing and fleeing as a dynamic defense: implications for the control of defensive behavior. Neurosci Biobehav Rev 29:1181-1191. CrossRef Medline

English AW, Tigges J, Lennard PR (1985) Anatomical organization of long ascending propriospinal neurons in the cat spinal cord. J Comp Neurol 240:349-358. CrossRef Medline

Flynn JR, Graham BA, Galea MP, Callister RJ (2011) The role of propriospinal interneurons in recovery from spinal cord injury. Neuropharmacology 60:809-822. CrossRef Medline

Forssberg H (1979) Stumbling corrective reaction: a phase-dependent compensatory reaction during locomotion. J Neurophysiol 42:936-953. CrossRef Medline

Forssberg H, Grillner S, Rossignol S (1977) Phasic gain control of reflexes from the dorsum of the paw during spinal locomotion. Brain Res 132: 121-139. CrossRef Medline

Frigon A (2011) Interindividual variability and its implications for locomotor adaptation following peripheral nerve and/or spinal cord injury. Prog Brain Res 188:101-118. CrossRef Medline

Frigon A (2017) The neural control of interlimb coordination during mammalian locomotion. J Neurophysiol 117:2224-2241. CrossRef Medline

Frigon A, Rossignol S (2007) Plasticity of reflexes from the foot during locomotion after denervating ankle extensors in intact cats. J Neurophysio 98:2122-2132. CrossRef Medline

Frigon A, Rossignol S (2008a) Adaptive changes of the locomotor pattern and cutaneous reflexes during locomotion studied in the same cats before and after spinalization. J Physiol 586:2927-2945. CrossRef Medline

Frigon A, Rossignol S (2008b) Short-latency crossed inhibitory responses in extensor muscles during locomotion in the cat. J Neurophysiol 99:989 998. CrossRef Medline

Frigon A, Barrière G, Leblond H, Rossignol S (2009) Asymmetric changes in cutaneous reflexes after a partial spinal lesion and retention following spinalization during locomotion in the cat. J Neurophysiol 102:26672680. CrossRef Medline

Frigon A, Hurteau MF, Thibaudier Y, Leblond H, Telonio A, D'Angelo G (2013) Split-belt walking alters the relationship between locomotor phases and cycle duration across speeds in intact and chronic spinalized adult cats. J Neurosci 33:8559-8566. CrossRef Medline

Frigon A, D'Angelo G, Thibaudier Y, Hurteau MF, Telonio A, Kuczynski V, Dambreville C (2014) Speed-dependent modulation of phase variations on a step-by-step basis and its impact on the consistency of interlimb coordination during quadrupedal locomotion in intact adult cats. J Neurophysiol 111:1885-1902. CrossRef Medline

Fuwa T, Shimamura M, Tanaka I (1991) Analysis of the forelimb crossed extension reflex in thalamic cats during stepping. Neurosci Res 9:257269. CrossRef Medline

Guertin P, Angel MJ, Perreault MC, McCrea DA (1995) Ankle extensor group I afferents excite extensors throughout the hindlimb during fictive locomotion in the cat. J Physiol 487:197-209. CrossRef Medline

Haridas C, Zehr EP (2003) Coordinated interlimb compensatory responses to electrical stimulation of cutaneous nerves in the hand and foot during walking. J Neurophysiol 90:2850-2861. CrossRef Medline

Hildebrand M (1976) Analysis of tetrapod gaits: general considerations and symmetrical gaits. In: Neural control of locomotion (Herman RM, Grillner S, Stein PS, Stuart DG, eds), pp 203-236. New York: Plenum.

Hildebrand M (1989) The quadrupedal gaits of vertebrates. Bioscience 39: 766-775. CrossRef

Hishinuma M, Yamaguchi T (1989) Modulation of reflex responses during fictive locomotion in the forelimb of the cat. Brain Res 482:184-188. CrossRef Medline

Hof AL, Duysens J (2018) Responses of human ankle muscles to mediolateral balance perturbations during walking. Hum Mov Sci 57:69-82. CrossRef Medline

Hurteau MF, Thibaudier Y, Dambreville C, Chraibi A, Desrochers E, Telonio A, Frigon A (2017) Nonlinear modulation of cutaneous reflexes with increasing speed of locomotion in spinal cats. J Neurosci 37:3896-3912. CrossRef Medline

Juvin L, Le Gal JP, Simmers J, Morin D (2012) Cervicolumbar coordination in mammalian quadrupedal locomotion: role of spinal thoracic circuitry and limb sensory inputs. J Neurosci 32:953-965. CrossRef Medline 
Juvin L, Grätsch S, Trillaud-Doppia E, Gariépy JF, Büschges A, Dubuc R (2016) A specific population of reticulospinal neurons controls the termination of locomotion. Cell Rep 15:2377-2386. CrossRef Medline

Kuczynski V, Telonio A, Thibaudier Y, Hurteau MF, Dambreville C, Desrochers E, Doelman A, Ross D, Frigon A (2017) Lack of adaptation during prolonged split-belt locomotion in the intact and spinal cat. J Physiol 595:5987-6006. CrossRef Medline

LaBella LA, Niechaj A, Rossignol S (1992) Low-threshold, short-latency cutaneous reflexes during fictive locomotion in the "semi-chronic" spinal cat. Exp Brain Res 91:236-248. Medline

Lamont EV, Zehr EP (2006) Task-specific modulation of cutaneous reflexes expressed at functionally relevant gait cycle phases during level and incline walking and stair climbing. Exp Brain Res 173:185-192. CrossRef Medline

Lamont EV, Zehr EP (2007) Earth-referenced handrail contact facilitates interlimb cutaneous reflexes during locomotion. J Neurophysiol 98:433442. CrossRef Medline

Loeb GE (1993) The distal hindlimb musculature of the cat: interanimal variability of locomotor activity and cutaneous reflexes. Exp Brain Res 96:125-140. CrossRef Medline

Matsukawa K, Kamei H, Minoda K, Udo M (1982) Interlimb coordination in cat locomotion investigated with perturbation: I. Behavioral and electromyographic study on symmetric limbs of decerebrate and awake walking cats. Exp Brain Res 46:425-437. CrossRef Medline

Matthews PB (1986) Observations on the automatic compensation of reflex gain on varying the pre-existing level of motor discharge in man. J Physiol 374:73-90. CrossRef Medline

Miller S, Ruit JB, Van der Meché FG (1977) Reversal of sign of long spinal reflexes dependent on the phase of the step cycle in the high decerebrate cat. Brain Res 128:447-459. CrossRef Medline

Nieuwenhuijzen PH, Schillings AM, Van Galen GP, Duysens J (2000) Modulation of the startle response during human gait. J Neurophysiol 84:6574. CrossRef Medline

Pratt CA, Chanaud CM, Loeb GE (1991) Functionally complex muscles of the cat hindlimb: IV. Intramuscular distribution of movement command signals and cutaneous reflexes in broad, bifunctional thigh muscles. Exp Brain Res 85:281-299. CrossRef Medline

Prochazka A, Sontag KH, Wand P (1978) Motor reactions to perturbations of gait: proprioceptive and somesthetic involvement. Neurosci Lett 7:3539. CrossRef Medline

Quevedo J, Stecina K, Gosgnach S, McCrea DA (2005a) Stumbling corrective reaction during fictive locomotion in the cat. J Neurophysiol 94: 2045-2052. CrossRef Medline

Quevedo J, Stecina K, McCrea DA (2005b) Intracellular analysis of reflex pathways underlying the stumbling corrective reaction during fictive locomotion in the cat. J Neurophysiol 94:2053-2062. CrossRef Medline

Reed WR, Shum-Siu A, Onifer SM, Magnuson DS (2006) Inter-enlargement pathways in the ventrolateral funiculus of the adult rat spinal cord. Neuroscience 142:1195-1207. CrossRef Medline

Roseberry T, Kreitzer A (2017) Neural circuitry for behavioural arrest. Philos Trans R Soc Lond B Biol Sci 372:20160197. CrossRef Medline

Ruder L, Takeoka A, Arber S (2016) Long-distance descending spinal neurons ensure quadrupedal locomotor stability. Neuron 92:1063-1078. CrossRef Medline
Schillings AM, Van Wezel BM, Duysens J (1996) Mechanically induced stumbling during human treadmill walking. J Neurosci Methods 67:1117. CrossRef Medline

Schillings AM, van Wezel BM, Mulder T, Duysens J (2000) Muscular responses and movement strategies during stumbling over obstacles. J Neurophysiol 83:2093-2102. CrossRef Medline

Schillings AM, Mulder T, Duysens J (2005) Stumbling over obstacles in older adults compared to young adults. J Neurophysiol 94:1158-1168. CrossRef Medline

Schomburg ED, Roesler J, Meinck HM (1977) Phase-dependent transmission in the excitatory propriospinal reflex pathway from forelimb afferents to lumbar motoneurones during fictive locomotion. Neurosci Lett 4:249-252. CrossRef Medline

Schomburg ED, Meinck HM, Haustein J, Roesler J (1978) Functional organization of the spinal reflex pathways from forelimb afferents to hindlimb motoneurones in the cat. Brain Res 139:21-33. CrossRef Medline

Schomburg ED, Steffens H, Warneke G (1986) Functional organization of the spinal reflex pathways from forelimb afferents to hindlimb motoneurones in the cat: II. Conditions of the interneuronal connections. Brain Res 375:280-290. CrossRef Medline

Seki K, Yamaguchi T (1997) Cutaneous reflex activity of the cat forelimb during fictive locomotion. Brain Res 753:56-62. CrossRef Medline

Sherrington CS (1906) The integrative action of the nervous system. New Haven, CT: Yale UP.

Shimamura M, Akert K (1965) Peripheral nervous relations of propriospinal and spino-bulbo-spinal reflex systems. Jpn J Physiol 15:638-647. CrossRef

Shimamura M, Livingston RB (1963) Longitudinal conduction systems serving spinal and brain-stem coordination. J Neurophysiol 26:258-272. CrossRef Medline

Shimamura M, Kogure I, Wada S (1982) Reticular neuron activities associated with locomotion in thalamic cats. Brain Res 231:51-62. CrossRef Medline

Thibaudier Y, Frigon A (2014) Spatiotemporal control of interlimb coordination during transverse split-belt locomotion with 1:1 or 2:1 coupling patterns in intact adult cats. J Neurophysiol 112:2006-2018. CrossRef Medline

Thibaudier Y, Hurteau MF (2012) Sensory regulation of quadrupedal locomotion: a top-down or bottom-up control system? J Neurophysiol 108: 709-711. CrossRef Medline

Thibaudier Y, Hurteau MF, Telonio A, Frigon A (2013) Coordination between the forelimbs and hindlimbs is bidirectional, asymmetrically organized, and flexible during quadrupedal locomotion in the intact adult cat. Neuroscience 240:13-26. CrossRef Medline

Thibaudier Y, Hurteau MF, Dambreville C, Chraibi A, Goetz L, Frigon A (2017) Interlimb coordination during tied-belt and transverse split-belt locomotion before and after an incomplete spinal cord injury. J Neurotrauma 34:1751-1765. CrossRef Medline

Zehr EP, Loadman PM (2012) Persistence of locomotor-related interlimb reflex networks during walking after stroke. Clin Neurophysiol 123:796807. CrossRef Medline

Zehr EP, Komiyama T, Stein RB (1997) Cutaneous reflexes during human gait: electromyographic and kinematic responses to electrical stimulation. J Neurophysiol 77:3311-3325. CrossRef Medline 